\title{
THE BURRELL-OPTICAL-KEPLER-SURVEY (BOKS). I. SURVEY DESCRIPTION AND INITIAL RESULTS
}

\author{
John J. Feldmeier ${ }^{1}$, Steve B. Howell ${ }^{2}$, William Sherry ${ }^{3}$, Kaspar von Braun ${ }^{4}$, \\ Mark E. Everett ${ }^{5}$, David R. Ciardi ${ }^{4}$, Paul Harding ${ }^{6}$, J. Christopher Mihos ${ }^{6}$, \\ Craig S. Rudick ${ }^{6}$, Ting-Hui LeE ${ }^{7}$, Rebecca M. Kutsko ${ }^{1}$, and Gerard T. van Belle ${ }^{8}$ \\ ${ }^{1}$ Department of Physics and Astronomy, Youngstown State University, Youngstown, OH 44555, USA; jjfeldmeier@ysu.edu \\ ${ }^{2}$ National Optical Astronomy Observatories, Tucson, AZ 85726, USA \\ ${ }^{3}$ National Solar Observatory, Tucson, AZ 85726, USA \\ ${ }^{4}$ NASA Exoplanet Science Institute, California Institute of Technology, Pasadena, CA 91125, USA \\ ${ }^{5}$ Planetary Science Institute, Tucson, AZ 85719, USA \\ ${ }^{6}$ Department of Astronomy, Case Western Reserve University, Cleveland, OH 44106, USA \\ ${ }^{7}$ Department of Physics and Astronomy, Western Kentucky University, Bowling Green, KY 42101, USA \\ ${ }^{8}$ European Southern Observatory, 85748 Garching, Germany \\ Received 2010 August 20; accepted 2011 March 13; published 2011 May 24
}

\begin{abstract}
We present the initial results of a 40 night contiguous ground-based campaign of time series photometric observations of a $1.39 \mathrm{deg}^{2}$ field located within the NASA Kepler Mission field of view. The goal of this pre-launch survey was to search for transiting extrasolar planets and to provide independent variability information of stellar sources. We have gathered a data set containing light curves of 54,687 stars from which we have created a statistical sub-sample of 13,786 stars between $14<r<18.5$ and have statistically examined each light curve to test for variability. We present a summary of our preliminary photometric findings including the overall level and content of stellar variability in this portion of the Kepler field and give some examples of unusual variable stars found within. We present a preliminary catalog of 2,457 candidate variable stars, of which 776 show signs of periodicity. We also present three potential exoplanet candidates, all of which should be observable by the Kepler mission.
\end{abstract}

Key words: binaries: eclipsing - planetary systems - techniques: photometric

Online-only material: color figures, machine-readable and VO tables

\section{INTRODUCTION}

Stellar variability studies provide critical access to a number of astronomically significant properties including rotation rates, eclipsing binaries, and pulsations. Aside from offering insight into the nature of the stars themselves, statistical studies conducted on large samples of field and cluster stars broaden our understanding of stellar evolution and can assist in the search for exoplanets. Variability surveys that endeavor to find variations with intermediate periods of days to weeks studying objects such as short period eclipsing binaries and planetary transits, benefit from dedicated long-term, high cadence observations (Howell 2008; von Braun et al. 2009a, and references therein).

NASA's Kepler Mission (Borucki et al. 2010b), which was launched in 2009 March, is conducting a transit search in Cygnus with the goal of finding Earth-like planets orbiting in the habitable zones of Sun-like stars. The signatures of transits of this nature would have relatively small depths, making the inherent variability of the host star even more relevant. The success of this mission partially depends on an accurate characterization of the stellar variability in the field. To that end, the Burrell-Optical-Kepler-Survey (BOKS) was designed to determine the level and type of stellar variability in a small $(\approx 1 \%)$ subsection of the Kepler field. As an added goal, we can assess the frequency of close-in Jovian-type planets (the so-called hot Jupiters) in the same field and allow for a comparison of ground-based and Kepler-based transit surveys. A number of other hot Jupiters were discovered via groundbased surveys (O'Donovan et al. 2006; Pál et al. 2008; Bakos et al. 2010) prior to Kepler's launch and Kepler itself has already discovered many additional exoplanets (Borucki et al. 2010c;
Koch et al. 2010; Dunham et al. 2010; Latham et al. 2010; Jenkins et al. 2010; Borucki et al. 2010a; Steffen et al. 2010). Further comparisons of ground-based and space-based transit candidates would be extremely beneficial due to the high quality light curves that Kepler can provide. In particular, since the Kepler Mission must make numerous selection cuts in order to achieve mission objectives (Batalha et al. 2010), there is a tendency to avoid fainter target stars that may have detectable hot Jupiters, but may not be suitable for Earth-sized transit searches. By identifying additional hot Jupiter candidates, and then having Kepler undertake follow-up observations, we may be able to study detailed properties of these systems and characterize them in exquisite detail. This has already been done for one pre-launch hot Jupiter candidate, HAT-P-7, and there are indications that the extrasolar planet in this system is gravitationally distorting the host star (Welsh et al. 2010).

This paper introduces the properties of BOKS and gives a summary of the data reduction and analysis of the survey. In Section 2, we present our observing strategy and a summary of our observations. We outline our data reduction techniques and discuss the observational window function of our survey in Section 3. We present the object detection, photometry, and astrometry in Section 4. Finally, in Section 5 we discuss the initial results of our variability survey and of the search for exoplanets in the BOKS field, specifically those of the "hot Jupiter" variety. We review our conclusions in Section 6. There are a number of other scientific projects planned for the BOKS data, such as the comparison of stellar field to cluster variability, cataloging the many variable stars found in this survey, and searching for any moving objects. These results will be discussed in future papers. We plan to submit all of the 
BOKS data to the NASA/IPAC/NExScI Star and Exoplanet Database $^{9}$ (von Braun et al. 2009b), where it can be of service to the entire astronomical community.

\section{OBSERVATIONS}

In order to maximize the scientific benefits from this survey, we chose our scientific field under a number of constraints. First, besides being located within the Kepler field our field ideally should have a large number of stars, as this will improve our chances to find extrasolar planets and find other interesting objects. Second, given that our ground-based imager has relatively large pixels in angular size, the field must not be so close to the Galactic plane that photometric crowding would be a major factor. Third, in order to compare our data against numerous stellar cluster variability surveys, such as UStAPS (Hood et al. 2005), EXPLORE/OC (von Braun et al. 2005), PISCES (Mochejska et al. 2006), and STEPSS (Burke et al. 2006), we decided on a field that had both field stars and an open cluster within it, so that we could compare the variability properties of both stellar populations simultaneously. In order to find the optimal field, we first pre-imaged a number of candidate fields on 2006 April 24. After visual inspection of all of the preimages, we made a determination of the field that best matched our conflicting criteria.

Our final selected target field of view covered $1.39 \mathrm{deg}^{2}$ in the constellation of Cygnus and was centered on the open cluster NGC 6811 (R.A. $=19^{\mathrm{h}} 37^{\mathrm{m}} 17^{s}$, decl. $=+46^{\mathrm{d}} 23^{\mathrm{m}} 18^{\mathrm{s}}$; WEBDA $^{10}$ ). Our field is completely contained within the Kepler field, and the BOKS field is located on channels 63, 47, 23, and 39 of the Kepler imager in the Spring, Summer, Fall, and Winter seasons, respectively. ${ }^{11}$ Our photometric survey began on 2006 September 1 and ended on 2006 October 10, consisting of 40 nights in all. We observed with the Case Western Reserve University $0.61 \mathrm{~m}$ Burrell Schmidt telescope (hereafter the Burrell), located at Kitt Peak National Observatory. One advantage of a dedicated observatory for this survey is that we could observe for a large number of consecutive nights. Many researchers (Pont et al. 2006; Beatty \& Gaudi 2008; von Braun et al. 2009a) have shown that the total duration of a photometric survey is crucial in maximizing transit detection efficiency in the presence of statistically correlated ("red") noise.

The imager used for this survey was a SITe back-illuminated $2 \mathrm{k} \times 4 \mathrm{k}$ CCD with 15 micron (1.45 arcsec) pixels, run by a Leach version 2 controller (Leach et al. 1998), and two output amplifiers. The long axis of the CCD was oriented east/ west. We observed primarily in the Sloan Digital Sky Survey (SDSS) $r$-band filter, but we also obtained occasional Johnson $V$-band images of the field of view in order to obtain two-color information and to allow for cross-comparison between our data and other photometric catalogs. In particular, we compared our photometry to that found from the Kepler Input Catalog (KIC). ${ }^{12}$

Observations were ongoing during any weather conditions where stars were visible on the sky, and it was safe to operate the telescope. As a result, the BOKS data have large variations in seeing, transparency, and night sky brightness. Of the 40

\footnotetext{
9 Located at http://nsted.ipac.caltech.edu/.

10 The WEBDA database, developed by J.-C. Mermilliod, can be found at http://www.univie.ac.at/webda/.

11 Full Frame Images (FFIs) of the Kepler field for each observing season can be found at http://archive.stsci.edu/kepler/ffi_display.php.

12 Version 10 of the KIC is available at http://archive.stsci.edu/kepler/kic10/ search.php. In some cases, we used the 7 th and 8 th versions of the KIC for steps in our analysis. These will be referred to as KIC78 in the text.
}

nights of observing, 13 were completely lost to weather, leaving 27 nights of potential data. Nearly all of our $r$-band and $V$ integrations were $180 \mathrm{~s}$ in duration, with the exception of 31 images taken on night 14 that had exposure times of $300 \mathrm{~s}$. The CCD readout time was $45 \mathrm{~s}$ in length. A total of $1,924 r$ images and $10 \mathrm{~V}$ images were taken over the entire run. The SITe CCD gain was fixed at 2 electrons per ADU, each pixel had a full well capacity of at least 100,000 electrons, and the read noise was 12 electrons. We note here that while the CCD pixel scale is fairly large compared to typical CCD imagers, studies (J. J. Feldmeier et al. 2011, in preparation) have shown that millimagnitude relative photometry is possible, even with such large pixels. Table 1 provides a summary log of our observations and Figure 1 shows a graphical representation of the number of exposures throughout the survey. An $r$-band exposure of BOKS, created from co-adding the first 24 images from our survey, is shown in Figure 2.

With the help of the American Association of Variable Star Observers (AAVSO), we also arranged to have bright variable stars photometrically monitored in the field at the same time as BOKS was underway. The preliminary results from this independent photometric survey are discussed in Henden et al. (2006), and a more careful comparison will be discussed in a future paper. ${ }^{13}$

\section{DATA REDUCTION}

Although the data reduction of BOKS is relatively straightforward, the large number of images and the need to ensure highly precise relative photometry demands some careful attention. We therefore began our CCD reductions as follows. Since our imaging observations were obtained using the dual amplifier mode with the SITe CCD, we first combined the two amplifier readouts into single images using IRAF's ${ }^{14}$ mscred.mkmsc task. The resulting images were then merged, trimmed, and overscan subtracted using the ccdred.ccdproc task.

We obtained approximately ten bias and five twilight flatfield frames per night, which were used on the respective night's images using IRAF's ccdred package after they were checked for unwanted features such as bright stars in the flat fields or amplifier noise in the bias frames. If any such features were present, the corresponding bias or flat-field frames were discarded. We created nightly master bias frames, but due to the presence of dust grains on the dewar window and/or filter, which changed positions between nights, we did not create a master flat for the entire run.

After the data had been processed, we inspected the entire data set for quality issues. We determined basic parameters of each image such as the median sky level and the median seeing in order to verify that our data were suitable for use. To determine the median sky level, we used the IRAF task imstatistics in iterative mode. The median seeing of each image was calculated by applying the imexam task on 150 bright, unsaturated stars on each image. Each star was fit using a Gaussian function, and the median of all of the derived FWHM values was adopted for the median seeing for each image.

After applying the photometric zero point (Section 4.3), we present the median sky brightness and median seeing for BOKS

\footnotetext{
13 The AAVSO NGC 6811 campaign information can be found at http://www.aavso.org/news/ngc6811.shtml.

14 IRAF is distributed by the National Optical Astronomy Observatory, which is operated by the Association of Universities for Research in Astronomy, Inc., under cooperative agreement with the National Science Foundation.
} 
Table 1

Observing Log Summary

\begin{tabular}{|c|c|c|c|c|c|}
\hline Night & JD & Hours Used & $\mathrm{N}_{\text {images }}$ & Airmass Range & Notes \\
\hline 1 & 2453980 & 1.68 & 25 & $1.06 \cdots 1.24$ & \\
\hline 2 & 2453981 & 0.00 & $\ldots$ & $\ldots$ & Unusable due to weather \\
\hline 3 & 2453982 & 0.00 & $\ldots$ & $\ldots$ & Unusable due to weather \\
\hline 4 & 2453983 & 0.00 & $\ldots$ & $\ldots$ & Unusable due to weather \\
\hline 5 & 2453984 & 0.00 & $\ldots$ & $\ldots$ & Unusable due to weather \\
\hline 6 & 2453985 & 0.00 & $\ldots$ & $\ldots$ & Unusable due to weather \\
\hline 7 & 2453986 & 0.00 & $\ldots$ & $\ldots$ & Unusable due to weather \\
\hline 8 & 2453987 & 0.00 & $\ldots$ & $\ldots$ & Unusable due to weather \\
\hline 9 & 2453988 & 0.42 & 6 & $1.19-1.25$ & \\
\hline 10 & 2453989 & 4.68 & 56 & $1.03-1.71$ & \\
\hline 11 & 2453990 & 0.00 & $\ldots$ & $\ldots$ & Unusable due to weather \\
\hline 12 & 2453991 & 0.00 & $\ldots$ & $\ldots$ & Removed- CCD condensation \\
\hline 13 & 2453992 & 0.00 & $\ldots$ & $\ldots$ & Removed- variable clouds \\
\hline 14 & 2453993 & 5.96 & 69 & $1.04-2.16$ & \\
\hline 15 & 2453994 & 5.87 & 81 & $1.04-2.08$ & \\
\hline 16 & 2453995 & 6.56 & 95 & $1.04-2.51$ & \\
\hline 17 & 2453996 & 6.33 & 92 & $1.03-2.82$ & \\
\hline 18 & 2453997 & 6.95 & 98 & $1.04-3.01$ & \\
\hline 19 & 2453998 & 0.00 & $\ldots$ & $\ldots$ & Removed- CCD electronics \\
\hline 20 & 2453999 & 2.63 & 38 & $1.04-1.13$ & \\
\hline 21 & 2454000 & 1.79 & 24 & $1.04-1.05$ & \\
\hline 22 & 2454001 & 6.54 & 86 & $1.04-2.55$ & \\
\hline 23 & 2454002 & 0.00 & $\ldots$ & $\ldots$ & Removed- variable clouds \\
\hline 24 & 2454003 & 6.64 & 91 & $1.04-2.77$ & \\
\hline 25 & 2454004 & 6.73 & 93 & $1.04-2.70$ & \\
\hline 26 & 2454005 & 6.60 & 93 & $1.04-2.72$ & \\
\hline 27 & 2454006 & 6.68 & 96 & $1.04-2.84$ & \\
\hline 28 & 2454007 & 5.86 & 84 & $1.04-2.08$ & \\
\hline 29 & 2454008 & 6.25 & 90 & $1.04-2.45$ & \\
\hline 30 & 2454009 & 4.75 & 66 & $1.04-1.58$ & \\
\hline 31 & 2454010 & 5.76 & 78 & $1.03-2.18$ & \\
\hline 32 & 2454011 & 5.13 & 57 & $1.03-1.97$ & \\
\hline 33 & 2454012 & 5.28 & 73 & $1.03-2.21$ & \\
\hline 34 & 2454013 & 0.00 & $\ldots$ & $\ldots$ & Unusable due to weather \\
\hline 35 & 2454014 & 0.00 & $\ldots$ & $\ldots$ & Unusable due to weather \\
\hline 36 & 2454015 & 5.61 & 74 & $1.03-2.59$ & \\
\hline 37 & 2454016 & 0.00 & $\ldots$ & & Removed- lunar background \\
\hline 38 & 2454017 & 0.00 & $\ldots$ & & Unusable due to weather \\
\hline 39 & 2454018 & 0.00 & $\ldots$ & & Unusable due to weather \\
\hline 40 & 2454019 & 0.00 & $\ldots$ & & Unusable due to weather \\
\hline
\end{tabular}

in Figure 3. As would be expected from a telescope run of this length, these properties varied substantially as differing lunar phases and weather patterns occurred. After this analysis, we removed night 37 's data due to very high sky levels by the nearly full moon. Next, we visually inspected each image for quality issues. From this process, we found that night 12 suffered from condensation on the CCD dewar window, leaving a circular distortion feature on approximately $17 \%$ of the area of each image. Night 19 suffered from CCD electronics issues on one of the two CCD amplifiers. In both of these cases, many of the stars remain unaffected on each image. However, to be conservative in this initial study, we have removed the entire night's data from further consideration. After our initial light curve analysis (discussed in Section 5), we found that two additional nights, nights 13 and 23, had extremely variable clouds (with such a large angular field, even the ensemble photometry method discussed in Section 4 can fail if the clouds are variable enough; J. J. Feldmeier et al. 2011, in preparation). Although some of the exposures on these nights should be acceptable for our scientific goals, we again chose to be conservative and removed the entire night's data from consideration. This left us with imaging data from 22 different nights over the span of the survey and a total of $1,565 r$-band images that could be used for potential variability studies.

For a planetary transit survey, understanding the observational window function gives crucial insight into the survey completeness and sensitivity to periodic variability. Normally, the window function is defined as the probability that a planetary transit is detected in a given data set, as a function of planetary orbital period (von Braun et al. 2009a). For BOKS, we calculated the approximate observable window function in the following manner: we simulated planetary transits from periods ranging from 0.5 to 30 days and divided each period into 10,000 phases. Given the starting and ending times of the 22 nights of observations, we then determined the probability of observing a transit with that period over all phases. The results of this analysis are plotted in Figure 4. As with all time-limited photometric surveys, we are most sensitive to short period transits, and our ability to detect transits decreases with transit period. Since our survey is ground-based, the characteristic aliasing period of integer days is also strongly present in our data. We should note that this window function deals with temporal sampling only and 


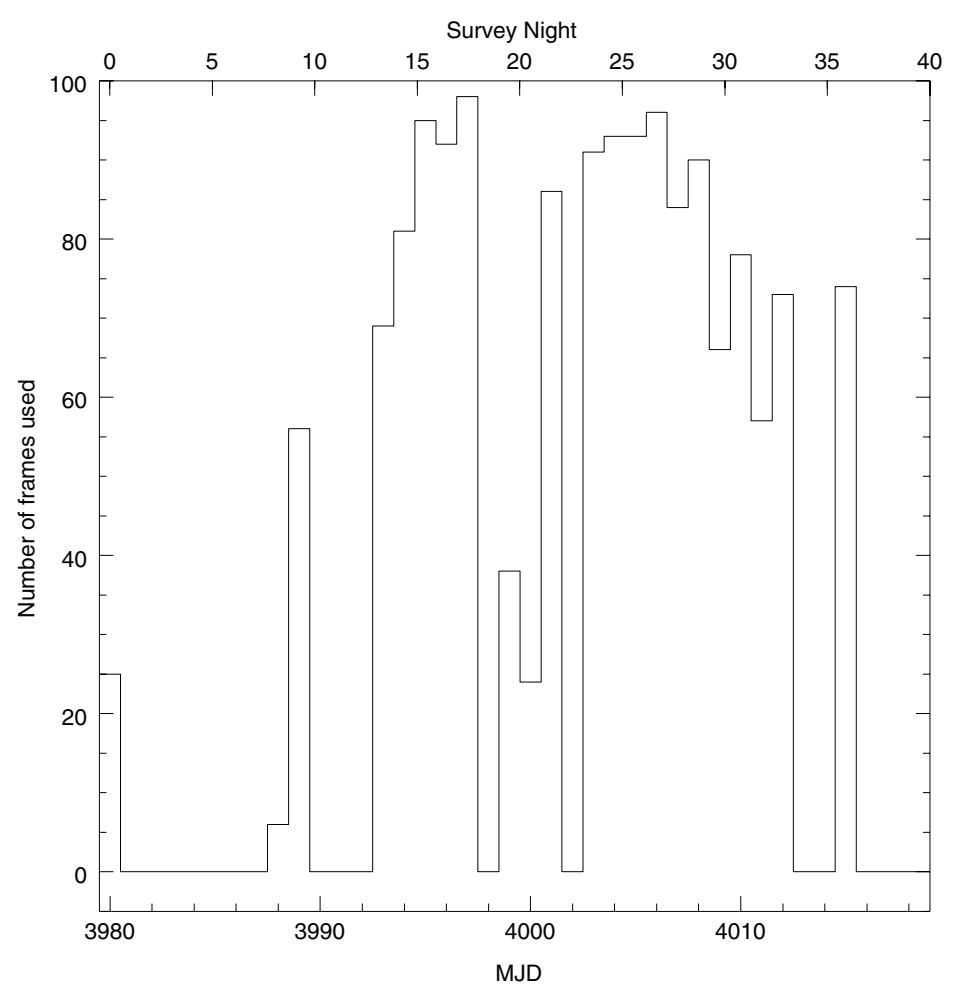

Figure 1. Number of BOKS images taken as a function of survey time. Due to the effects of thick clouds, and occasional high winds, the numbers of nightly frames taken vary significantly throughout the survey.

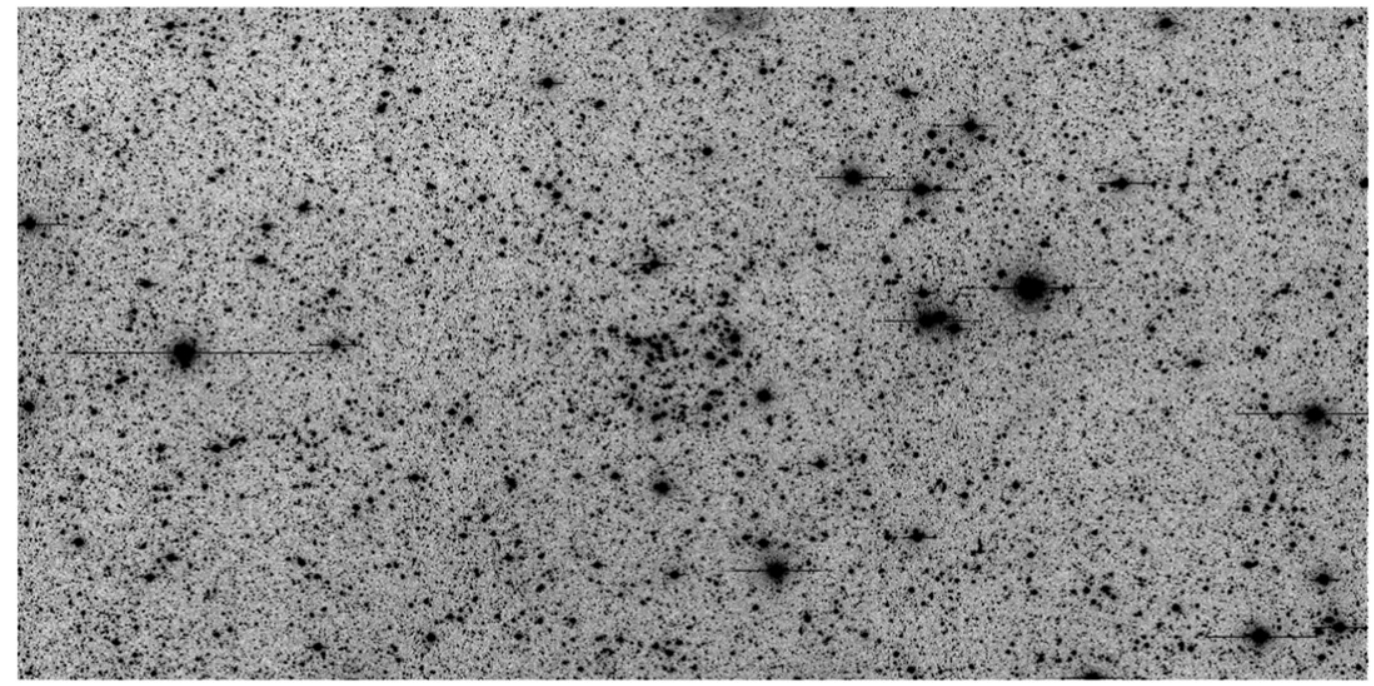

Figure 2. Image of the BOKS field, created by combining the first 24 images of the survey. This image is approximately 101.5 by 49.5 in size. North is up and east is to the left. The open cluster NGC 6811 , whose center is located at $\alpha=19^{\mathrm{h}} 37^{\mathrm{m}} 17^{\mathrm{s}}, \delta=+46^{\mathrm{d}} 23^{\mathrm{m}} 18^{\mathrm{s}}$ (WEBDA database), is clearly visible in the center of the image.

likely to be an overestimate: it does not take into account the effects of differing transparency, seeing, and sky brightness on the detectability of transits. It also does not take into account the effects of differing stellar and planetary radii on the detectability of transits. Finally, the effects of statistically correlated "red noise," which are likely to be significant, are not included in this calculation. We plan to perform extensive Monte Carlo simulations on these effects, which will be presented in a future paper. However, we note that BOKS has a significant advantage over many other ground-based transit surveys: if even one transit appears in our survey, we could, in principle, verify it with Kepler follow-up observations.

\section{OBJECT DETECTION, PHOTOMETRY, AND ASTROMETRY}

With our good quality data set finalized, we next focused our attention on finding all stellar sources in the BOKS field, and determining their magnitudes and positions throughout the survey period.

\subsection{Object Detection and Coordinate Transformations}

We next created a master image by combining six individual images from night 25 using the imcombine task. This master image was used to ensure that we detect all of the stellar sources 

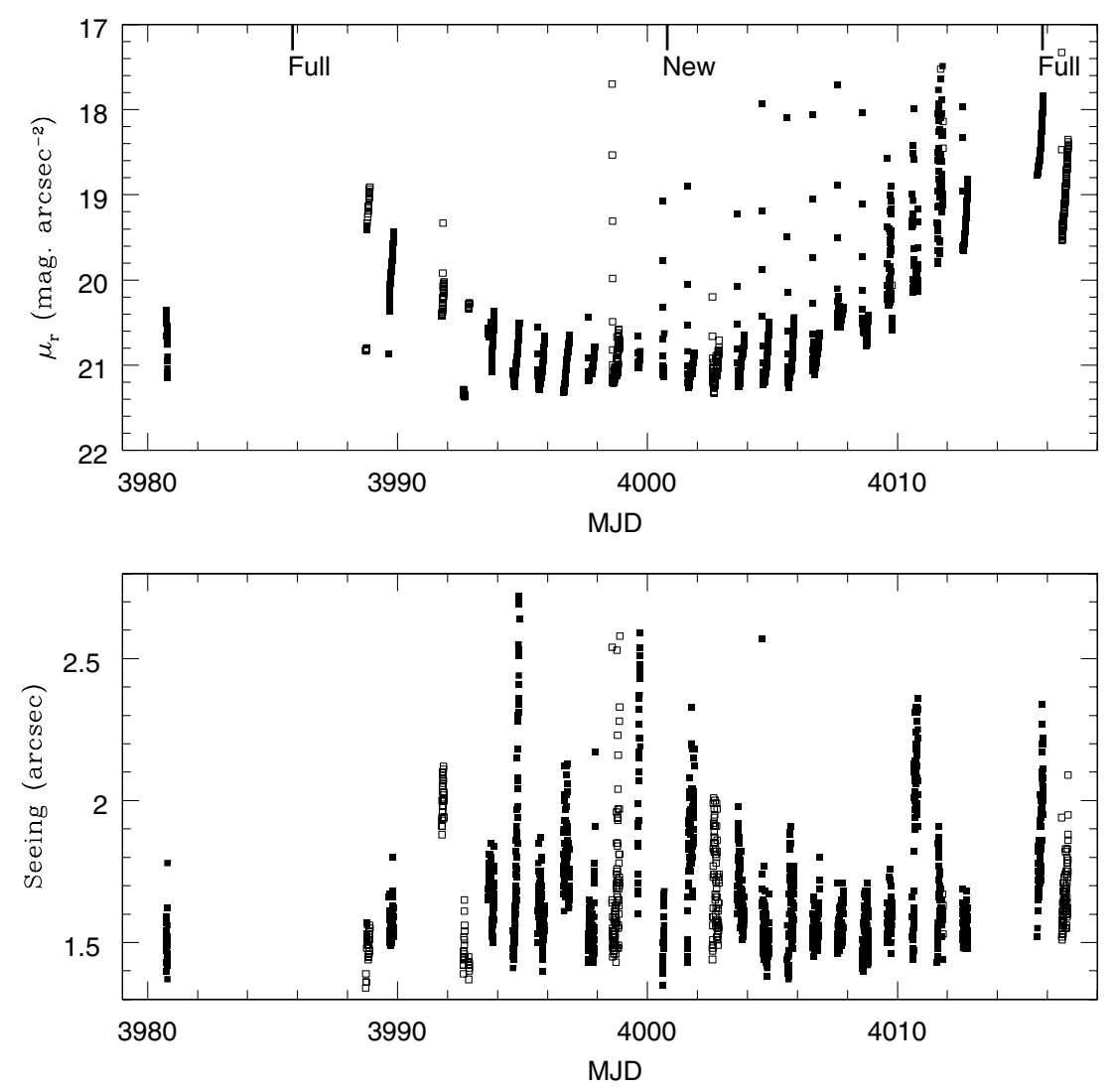

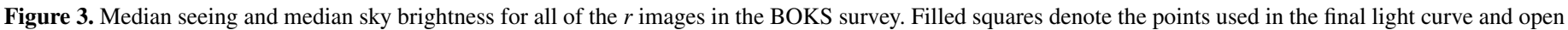

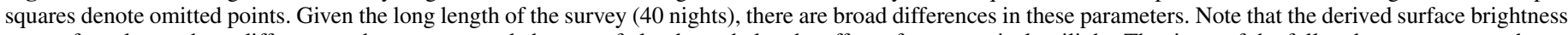

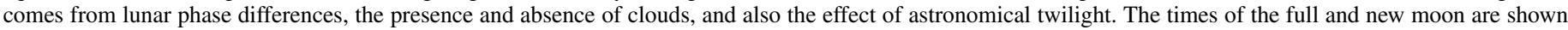

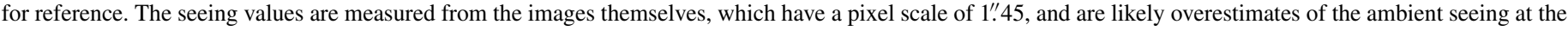
time of the observations.

in the frame and remove the possibility of radiation events contaminating our source catalog. We next created the master list of stars (point sources) by running the daof ind task within IRAF on the master image. We chose a threshold value for point-source detection of five times the standard deviation of the sky background. Given the large pixel scale of our data, we adjusted the sharphi value to 0.9 rather than the 1.2 that is normally adopted. All other data-independent parameters were left at their default values. We found a total of 56,354 sources that matched the daofind criteria we adopted. This initial list was then manually inspected to ensure that non-stellar objects were not included, such as diffraction spikes, radiation events, or objects on the extreme edges of the frame. This left a total of 54,687 objects for further study.

With the master list of coordinates determined, we then needed to re-identify each source on every frame of our survey. Rather than shift each image, which would lead to unacceptable uncertainties in the magnitudes due to interpolation, we instead re-determined the source coordinates for each individual image. To do this, we used a high quality image from the middle of night 25 as our positional reference image. To match stars in individual images to those found on the reference image, we split each image into eight rectangular subsections $(512 \times 512$ pixels $)$. For each subsection of each image, we summed a group of rows and group of columns to create a pair of one-dimensional arrays that contained the peak counts at the row or column location of each star. We then used the Fourier transform between pairs of these arrays taken from different images to locate translational shifts between each subsection of the images. These shifts were applied to the master coordinate list to locate each star on individual images. The Burrell tracked fairly well overall but in periods of cloudy conditions, when the autoguider was unable to hold the tracking steady, the spatial shifts could be up to 1-2 arcmin. By dividing the image into subsections, we were able to accommodate most of the magnification and rotational differences between images in our final astrometric coordinate solution.

\subsection{Aperture Photometry and Ensemble Correction}

We performed aperture photometry using the phot task within IRAF's noao.digiphot.daophot package (Stetson 1987). We experimented with five separate photometry apertures, from very small radii (1 pixel) to large radii (5 pixels), to span the range of stellar brightness and seeing changes over the observed field of view. After inspection of the output light curves, we selected two distinct aperture values for our work (3 and 4 pixels) based on their small level of scatter around the median magnitude of a representative light curve sample. These two aperture values, which correspond to 4.35 arcsec and 5.8 arcsec with a sky annulus of 11.6-33 arcsec, gave good results and we used the smaller aperture for our final light curve set.

Once the raw photometry files were created, we then needed to account for the effects of seeing, transparency, and airmass for each star. To do this, we adopted a local ensemble photometry technique, where we used a local set of bright stars that are photometrically constant to determine changes in these 


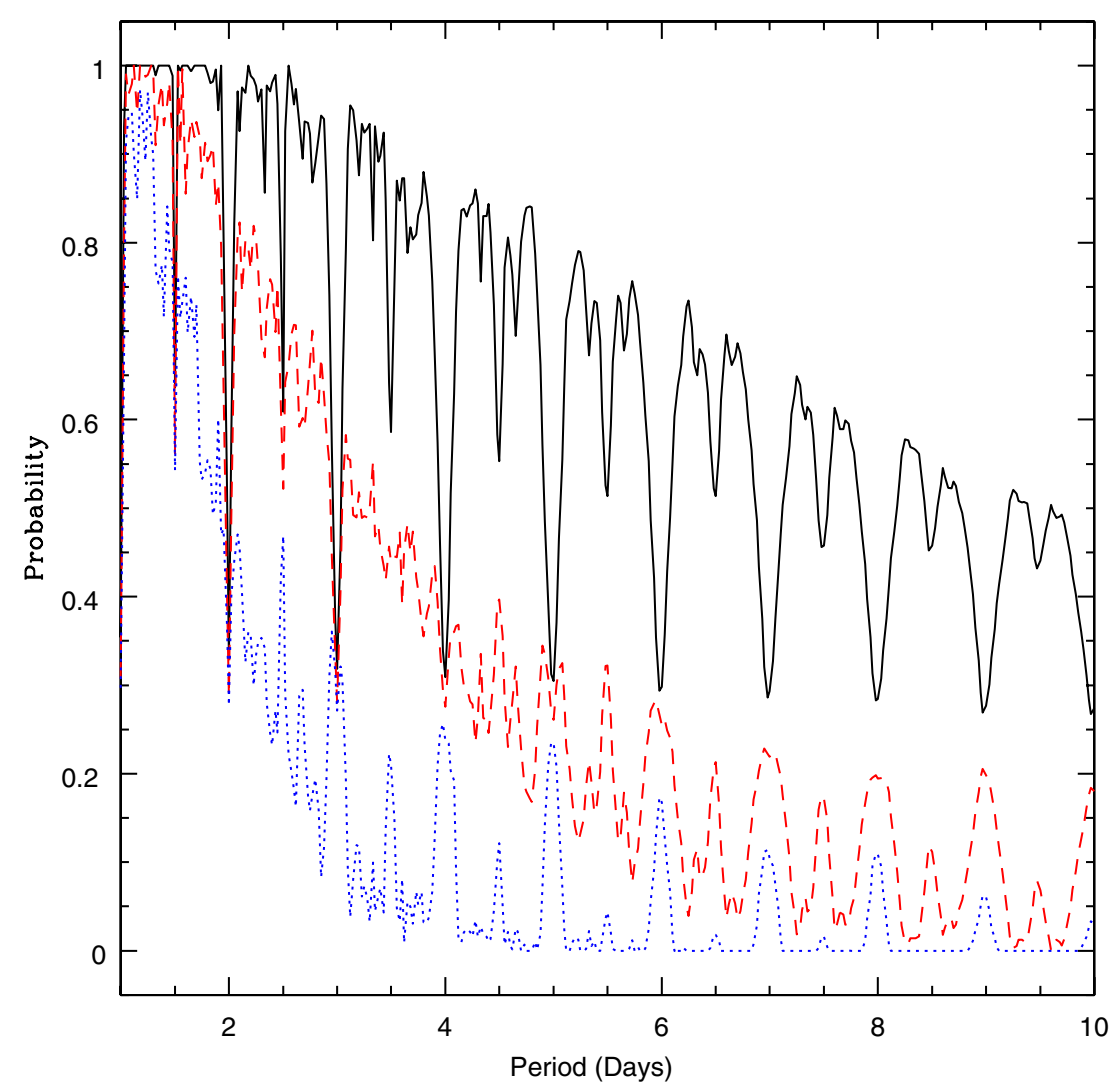

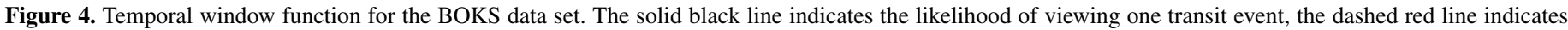

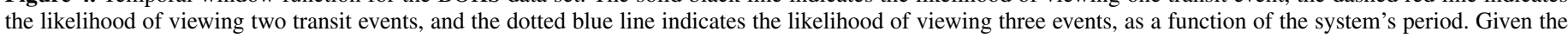

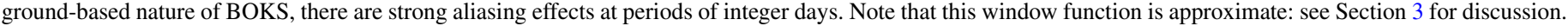

(A color version of this figure is available in the online journal.)

parameters. The algorithms are discussed in detail by Everett et al. (2002) and Everett \& Howell (2001); we will briefly outline them here.

First, we divided each image into $8 \times 4$ square regions with a size of $500 \times 500$ pixels (corresponding to 725 arcsec on the side). This size was chosen to allow a sufficient number of ensemble stars to be present in the individual regions and to optimize our sampling of positional dependence of photometric effects such as variable point-spread functions, color terms, or focus gradients. For a star to be an acceptable ensemble star, it must fulfill certain quality criteria. Specifically, (1) the star must be present in all frames, (2) it must have a photometrically constant light curve $\left(\chi^{2}<3\right.$.), (3) it must be bright enough so that photon noise is negligible (average flux must be greater than 50,000 ADU, corresponding to a signal-to-noise ratio of 316 or better), and (4) have no close-by stellar companions that would interfere with the light curve in poor seeing conditions (no stars within 5 pixels that are within 5 mag of the ensemble candidate's magnitude). We created an initial list of ensemble stars by conducting an automated search through the stars in each region. After this automated preselection, the ensemble stars were inspected by eye to eliminate stars that appeared to have signs of residual variability compared to the remainder of the ensemble stars. A total of 688 stars with $r$ magnitudes varying between 14.3 and 16.6 were used in the final ensemble.

After the final list of ensemble stars was created, the relative photometry procedure was rerun using only the cleaned sample of stars. If a region had fewer than 10 ensemble stars, we combined it with a neighboring one to create a larger region. The exact calculation of relative photometric offsets for the individual regions was performed by a custom written routine based on Everett et al. (2002). An example of these offsets for a single region is plotted in Figure 5. Due to the effects of airmass, seeing, and transparency, these offset values vary significantly over the survey length.

Due to the differing positions of each individual image, various stars have differing numbers of observations, with objects at the edges of the fields having fewer observations than objects near the center. To ensure a high quality set of light curves for study, we focus exclusively on light curves that have at least 1,000 photometric measurements. This left 32,806 sources for further analysis.

An unfortunate issue we found during the data reduction was the fact that the start time (HJD) for any given exposure listed in the image header was not sufficiently accurate for our purposes. The internal clock on the data recording computer, which was a Microsoft Windows PC running the Voodoo image acquisition software $^{15}$ at the telescope, was found to be imprecise and could not be used alone for exact timing purposes as we found that it drifted by up to several minutes over the course of a single night. As a result, we used the file creation times (recorded by a different clock on an internet time controlled Linux machine) as HJD "start time" information in our image headers. From some experimentation, we found that the header start time recorded is approximately $93 \mathrm{~s}$ after the true mid-exposure time in most cases. From comparing consecutive exposures throughout the survey, the uncertainty in this correction is approximately $1 \mathrm{~s}$. Consequently, the times we recorded in our light curves will

\footnotetext{
15 Located at http://www.astro-cam.com/.
} 


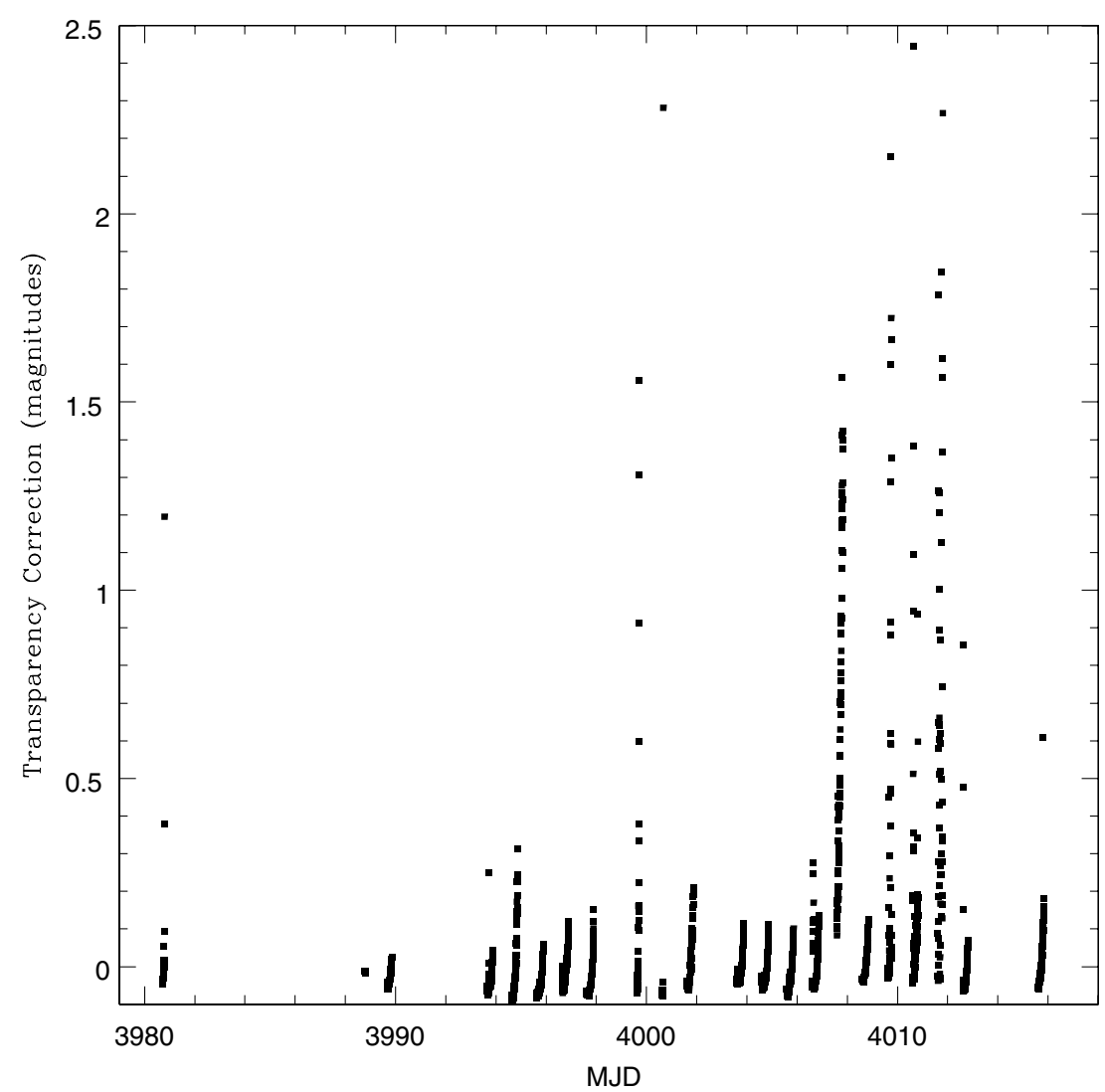

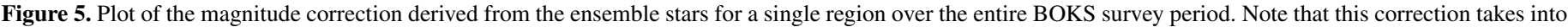

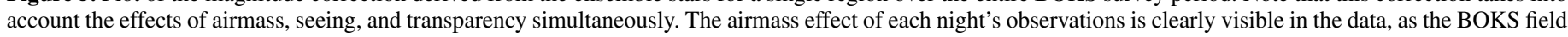
was close to zenith at the beginning of each night, and set as the night progressed.

be precise relative to each other, but our time zero point is not tied to UT or any other absolute time system to within several minutes. In the future, we plan to correct this by correlating our observations against the AAVSO and Kepler observations, which should allow us to reduce any time offsets.

\subsection{Photometric Zero Point}

The transformation from differential instrumental magnitudes to SDSS $r$ magnitudes for our stars was performed by comparing the magnitudes of the ensemble stars in each subregion to the corresponding $r$ stellar magnitudes listed in the KIC. The scatter in this comparison was approximately $0.1 \mathrm{mag}$, which we take as our systematic magnitude uncertainty for the BOKS survey. Given that we have minimal color information in our observations and our survey is primarily interested in searching for stellar variability, this amount of uncertainty is acceptable for our needs. For any individual object, however, one can derive a more accurate magnitude by directly comparing the object to the KIC values, which have a mean $r$ systematic uncertainty of less than 0.015 mag. ${ }^{16}$

\subsection{Astrometry}

We performed astrometry using the USNO-B1.0 catalog (Monet et al. 2003) and wcstools. ${ }^{17}$ In order to eliminate the effects of field rotation and distortion, we performed astrometry on the individual regions as described above, rather than on the

\footnotetext{
16 Photometric uncertainties for the KIC can be found at http://www.cfa.harvard.edu/kepler/kic/kicindex.html.

17 http://tdc-www.harvard.edu/software/wcstools/
}

field as a whole. Our internal uncertainties on our coordinates were approximately \pm 0 .' 5 right ascension and \pm 0 .' 3 declination. As an independent check on our astrometry, we compared our final astrometric catalog to that found from the Two Micron All Sky Survey (2MASS) Point Source Catalog (Skrutskie et al. 2006). Figure 6 shows the astrometric comparison between our BOKS astrometric solutions and those listed for common stars in the 2MASS catalog. The $1 \sigma$ coordinate offset is near $0{ }^{\prime \prime} 4$ both R.A. and decl. with a small but clear asymmetry across the field. Given that the pixel size of the Burrell is 1". 45 and that the wide field of the Burrell produces differential focus and refraction effects, this agreement is quite reasonable.

\section{LIGHT CURVE ANALYSIS}

With the light curves established for each star, we then began the search for photometric variability. To characterize the possible variable nature of our light curve sample and to search for possible exoplanet transits, we utilized OPTICSTAT, a custom-written statistical analysis package created by $\mathrm{M}$. Everett (discussed in Howell et al. 2005). OPTICSTAT returns several statistics related to stellar variability, including the reduced $\chi^{2}\left(\chi_{v}^{2}\right)$, the standard deviation, the probability that the star varies periodically, and the most likely period. Prior to our statistical light curve analysis, we removed the effects of obvious cosmic rays which will artificially increase the apparent flux as follows. If there were one or two consecutive points in the light curve that deviated from the mean magnitude by more than 3.5 times the standard deviation, those points were rejected during statistical tests. This might remove some signs of ultrashort variability, but since the exposures are $180 \mathrm{~s}$ in length and 


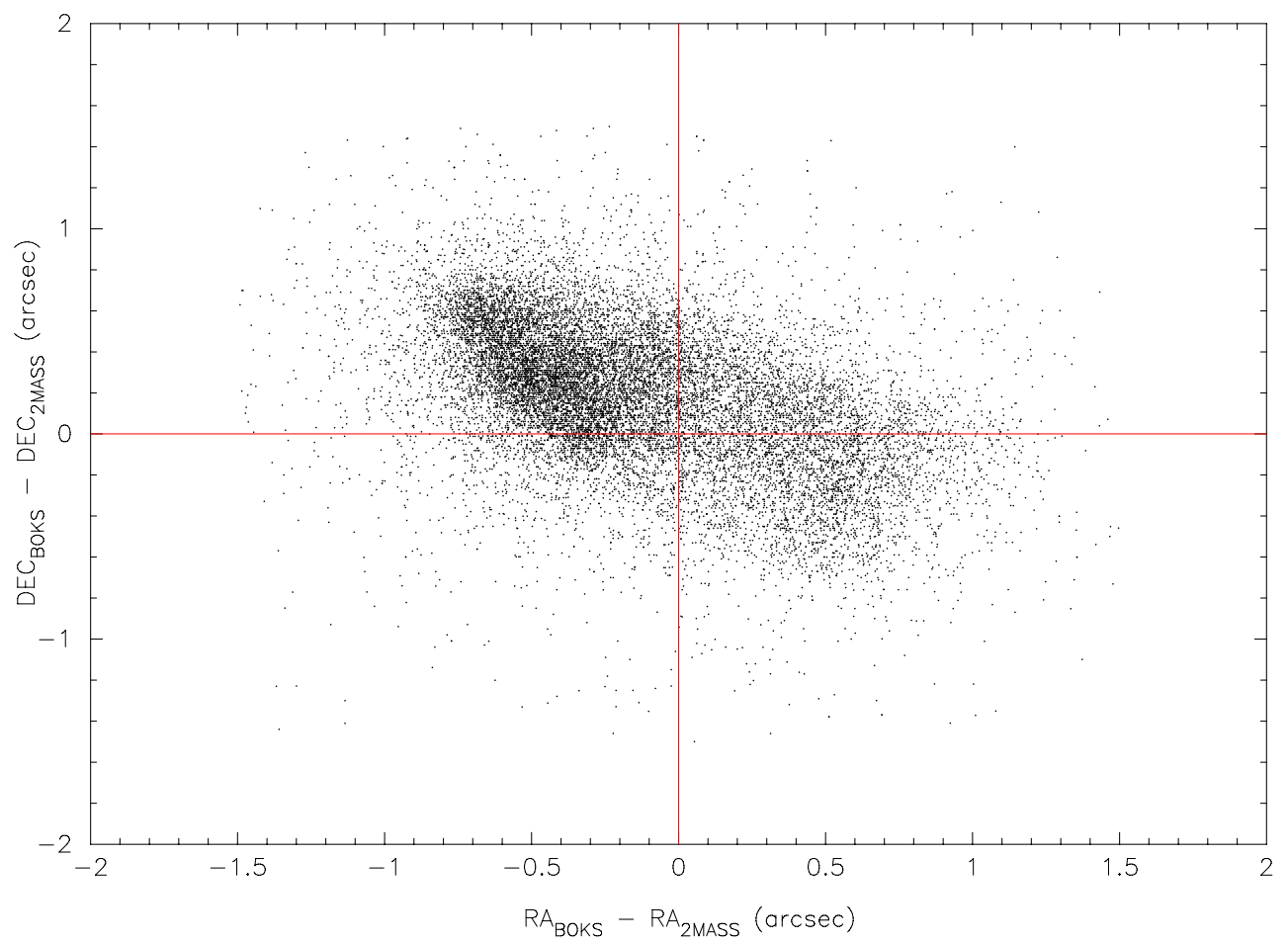

Figure 6. Deviation of the BOKS astrometric coordinates to the coordinates found from the 2MASS survey. These deviations should be compared to the pixel scale of the Burrell, which is 1". 45 .

(A color version of this figure is available in the online journal.)

the readout time of the CCD is $45 \mathrm{~s}$, the total scientific impact should be minimal.

To test each light curve for general variability, we fitted the light curve with its mean flux and then calculated the probability that the reduced $\chi_{v}^{2}$ statistic shows the data to be inconsistent with this mean flux. This test can easily fail, however, in the presence of systematic errors or uncertainties in the calculated magnitude errors we assign to the individual data points. To compensate, we adopted a more conservative threshold for the $\chi_{v}^{2}$ probability than our formal errors would dictate. When applied to the full light curve, we have adopted the criteria that point sources that have $\chi_{v}^{2} \geqslant 5$ are variable sources.

\subsection{Photometric Uncertainties}

In order to make a proper determination of variability for each star, we must determine the random and systematic uncertainties for our target stars. By splitting the entire BOKS field of view into 32 smaller sub-sections we were able to remove the vast majority of systematic variations in our light curves. However, some dispersion remains due to photometric uncertainties, small differences in color and properties between the ensemble stars and the target objects, and the statistically correlated ("red" noise) that is present in all ground-based transit surveys (Pont et al. 2006; Beatty \& Gaudi 2008; von Braun et al. 2009a).

To estimate our remaining photometric uncertainties for every light curve, we calculated a weighted average magnitude and the standard deviation of the entire light curve about this average. Figure 7 shows the standard deviation of our BOKS light curves as a function of their $r$ magnitude. As can be clearly seen, our brightest stars have a 4 mmag dispersion about the average magnitude, which sets our noise floor for this survey. For stars brighter than $r \simeq 14$, saturation becomes an issue with seeing changes making the exact magnitude of saturation somewhat imprecise.
As the apparent magnitude increases, the photometric errors also increase. Plotted in Figure 7 is an estimate of the photometric errors as a function of target flux (Everett \& Howell 2001):

$\sigma_{*}=1.0857 \frac{\sqrt{N_{*} \times g+n_{\text {pix }}\left[1+\left(n_{\text {pix }} / n_{\text {sky }}\right)\right]\left(N_{\text {sky }} \times g+R^{2}\right)}}{N_{*} \times g}$,

where $N_{*}$ is the number of ADUs from the star in the aperture, $g$ is the gain of the CCD in electrons ( $2 \mathrm{e}^{-}$per ADU), $n_{\text {pix }}$ is the number of pixels in the aperture, $n_{\text {sky }}$ is the number of pixels in the annulus around the aperture used to measure the sky flux, $N_{\text {sky }}$ is the flux in ADUs per pixel from the sky, and $R$ is the rms read noise of the CCD in electrons $\left(12 e^{-1}\right)$. For this comparison, we assumed the faintest value of the night sky brightness in our survey, which should give a lower bound to the true photometric uncertainties. As can be seen, this function is in good agreement with the lower edge of our error distribution. At the faint end of our photometry, near $r=19$, our uncertainties are $\sim 50$ mmag per observation, substantially larger than the 10-30 mmag precisions needed to find transiting extrasolar planets.

For completeness, we have also calculated the variability level expected from atmospheric scintillation alone (Young 1967; see also Young 1993a; Young 1993b; Badiali et al. 1994 for some important comments) in order to rule it out as a significant contributor of our highest precision photometric results. We find that the scintillation at unit airmass is approximately $0.3 \mathrm{mmag}$, about a factor of 15 lower than the photon noise from the brightest stars in our sample. This is an approximate value: Young (1993b) notes that the value can vary by up to a factor of two on a timescale of a few minutes. Nevertheless, given our measured photometric uncertainties, scintillation is not a major contributor to our error. 


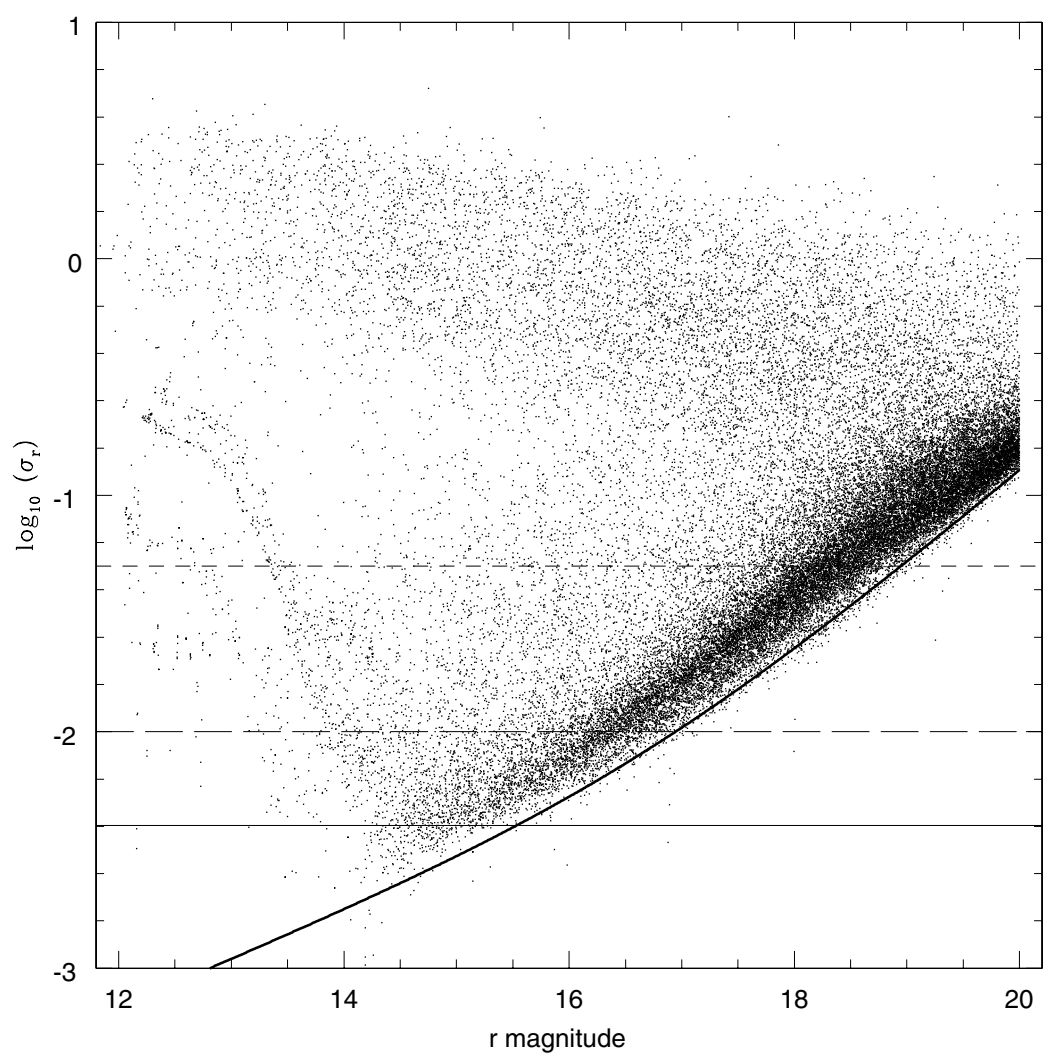

Figure 7. Standard deviation of the light curve of each star as a function of $r$ magnitude, as determined by OPTICSTAT, for all stars brighter than an $r$ magnitude of 20. Note that this plot includes every source, so variable stars will lie above the diagonal sequence that denotes the photometric error function. The upward bending of the points brighter than $r=14$. is due to saturation effects. The thick diagonal line denotes the expected photometric uncertainty for a source observed at new moon and should denote the lower edge of the true uncertainty distribution. The solid horizontal line shows a standard deviation of 4 mmag, the long horizontal line shows a standard deviation of $10 \mathrm{mmag}$, and the short dashed horizontal line shows a standard deviation of $50 \mathrm{mmag}$, for reference.

\subsection{Point-source Variability Statistics}

An important goal of BOKS is to determine the variability properties of stellar sources in general within one portion of the Kepler field. As mentioned previously, DAOFIND identified 56,354 point sources in at least one BOKS survey image. Of these, 32,806 point sources were observed at least 1,000 times within our survey. From this subset, 25,861 point sources are located within 2.5 arcsec of a source in the KIC78, and therefore we have additional photometric information. However, since the KIC78 data are non-contemporary with BOKS, any values derived from this comparison should be treated carefully. For statistical purposes, we excluded any source from the KIC78 that did not have a valid $r$ magnitude, even if it had measured magnitudes in other bands. This left 22,340 sources for further analysis.

We then determined a magnitude cutoff value for our statistical analysis of variability. Progressively fainter stars have larger photometric errors, are more likely to have poor quality light curves due to contamination from nearby bright stars and in conditions of poor seeing, and may have incorrect recentering by the aperture photometry algorithm, which can cause the aperture to recenter on a nearby brighter star. For these reasons, we restricted our variability statistics to the 13,786 stars brighter than $r=18.5$, which also lie within 2 .'5 of a source within the KIC78 and which have over 1000 data points. We hereafter refer to this subsample as the BOKS-KIC sample.

After applying the statistical tests from OPTICSTAT, we found 2,457 stars with $\chi_{v}^{2} \geqslant 5$ and $r<18.5$ in the BOKS-KIC sample. We note that this number is approximate, as the reduced $\chi^{2}$ is strongly affected by a number of systematic uncertainties, such as residual variability in our ensemble stars, color mismatches between the ensemble and the target stars, spatial structure in the extinction correction, and other effects. Additionally, photometric uncertainties and stellar variability applied to the KIC78 will move stars above and below the magnitude cutoff, creating an additional systematic uncertainty.

The variability fraction found $(\approx 18 \%)$ can be compared to other variability surveys using identical search techniques. In an earlier study, Everett et al. (2002) found a variability fraction of $17 \%$ over a five-day survey period using a similar telescope and sampling rate, but using a less strict variability criteria $\left(\chi_{v}^{2} \geqslant 3\right)$. In contrast, the variability study of NGC 2301 (Tonry et al. 2005; Howell et al. 2005; Sukhbold \& Howell 2009) found a much larger variability fraction (56\%) using an orthogonal transfer CCD observing mode over a 12 night run, and with substantially better photometric precision $(\approx 1.6 \mathrm{mmag})$.

Tonry et al. (2005) and Howell (2008) discussed a relation between the percentage of variable sources that will be found in any given photometric survey and that survey's photometric uncertainty. From the NGC 2301 results, the cumulative fraction of variable stars found, as a function of quartile variability, $x$ (the quartile variability is $\approx 1.5$ times smaller than the standard deviation), is ${ }^{18}$

$$
f(<x)=1-\frac{1.6 \mathrm{mmag}}{x} .
$$

\footnotetext{
18 Again, we note that in this paper we use $\chi^{2}>5$ while the previous studies used $\chi^{2}>3$, which would allow more low amplitude variables into the samples.
} 
Table 2

Nonperiodic Variable Candidates

\begin{tabular}{lcccccc}
\hline \hline $\begin{array}{l}\text { BOKS } \\
\text { ID }\end{array}$ & $\begin{array}{c}\text { KIC } \\
\text { ID }\end{array}$ & $\begin{array}{c}\text { R.A. } \\
(\mathrm{J} 2000)\end{array}$ & $\begin{array}{c}\text { Decl. } \\
(\mathrm{J} 2000)\end{array}$ & $\begin{array}{c}r \\
(\mathrm{mag})\end{array}$ & $\begin{array}{c}g-r \\
(\mathrm{mag})\end{array}$ & $\begin{array}{c}\text { Std. Deviation } \\
(\mathrm{mag})\end{array}$ \\
\hline 500 & 9652301 & 193225.376 & 462347.44 & 17.53 & 0.671 & 0.13 \\
593 & 9833939 & 193225.013 & 463947.88 & 15.86 & 0.471 & 0.45 \\
622 & 9893877 & 193224.968 & 464510.07 & 17.91 & 0.877 & 0.36 \\
648 & 9893880 & 193225.290 & 464314.87 & 17.49 & 0.576 & 0.18 \\
658 & 9713336 & 193226.869 & 462631.79 & 16.62 & 0.541 & 0.40 \\
670 & 9529529 & 193228.384 & 460853.23 & 17.49 & 0.812 & 0.06 \\
679 & 9833949 & 193225.848 & 464009.08 & 15.81 & 0.462 & 0.39 \\
739 & 9529537 & 193228.931 & 461111.50 & 18.33 & 0.796 & 0.10 \\
768 & 9529543 & 193229.390 & 460847.91 & 18.31 & 0.617 & 0.13 \\
844 & 9529545 & 193229.917 & 461130.64 & 17.85 & 0.582 & 0.09 \\
\hline
\end{tabular}

(This table is available in its entirety in machine-readable and Virtual Observatory (VO) forms in the online journal. A portion is shown here for guidance regarding its form and content.)

For a best precision of $4 \mathrm{mmag}$, like we have in the BOKS survey, the percentage of variable sources would be expected to be $\sim 40 \%$, substantially larger than what was actually detected. Some of this difference may be due to differing stellar populations: NGC 2301 is a young open cluster (250 Myr; Kim et al. 2001), in contrast to NGC 6811 (575 Myr; Luo et al. 2009), and the level of stellar activity on the main sequence may be significantly different between the two clusters. The amount of background and foreground contamination may vary significantly between the two surveys as well. Given the position of the BOKS field, it is likely that many of the stars are field stars and therefore have lower amounts of variability.

Of the 2,457 variable sources, 776 (32\% of all the variables or $\approx 6 \%$ of the total BOKS-KIC sample) were found to be periodic variable candidates (see Section 5.2.2). This is significantly larger than the results of Everett et al. (2002), who found a $14 \%$ periodic fraction in their survey, and Howell et al. (2005), who found a $13 \%$ periodic variable fraction in NGC 2301. We believe this is due to the substantially longer time coverage of BOKS, which should make it substantially easier for our search algorithm Section 5.2.2 to detect periodicity. The remaining variable stars appear to be non-periodic within the limits of our time sampling, photometric precision, and observational window. These non-periodic variable stars are presented in Table 2, with the corresponding photometric information from the KIC given. We caution that the standard deviations given for these light curves are likely to be an overestimate: any photometric residuals in the ensemble stars and the effect of radiation events can increase this value significantly.

There have been three other recent variability surveys of the regions of the BOKS field, all centered on the NGC 6811 open cluster (van Cauteren et al. 2005; Rose \& Hintz 2007; Luo et al. 2009). Unfortunately, the majority of the stars found to be variable by these surveys are saturated on the BOKS images. The three exceptions, stars V8 and V9 found by van Cauteren et al. (2005), and star V17 found by Luo et al. (2009), were also detected by BOKS, and all three were found to be periodic variable stars.

\subsubsection{Non-variable Stars}

Our variability analysis found 10,881 stars brighter than $r=18.0$ with no detected variability $\left(\chi_{v}^{2}<5\right)$. Figure 8 shows a color-magnitude diagram (CMD) of these stars. This CMD is typical of stellar fields within the galactic disk, showing

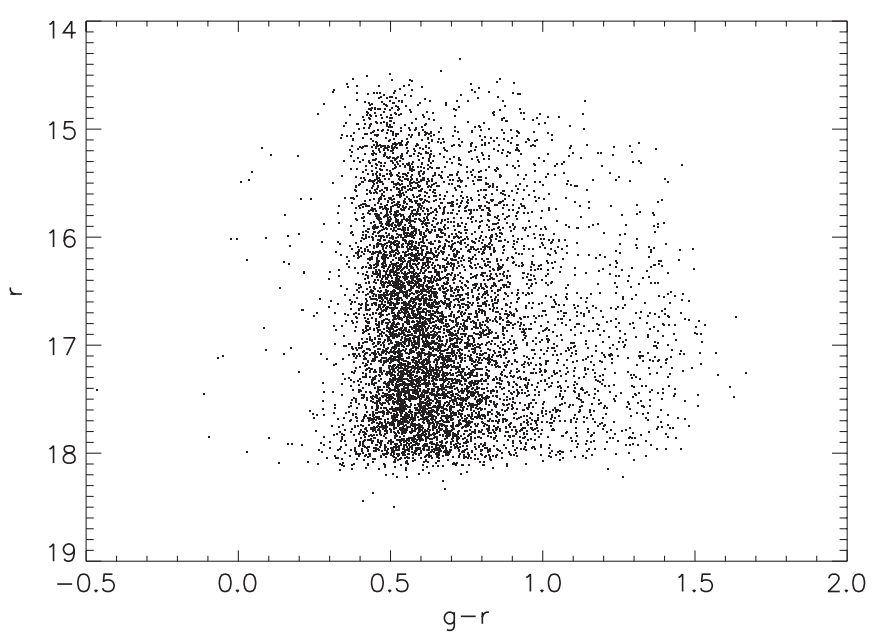

Figure 8. g-r CMD for the 10,881 non-variable stars detected with this survey that have entries in the Kepler Input Catalog. Note the single broad sequence of stars at $g-r \approx 0.5$, which corresponds to stars at the main-sequence turn-off for an old stellar population. This CMD can be compared to the much deeper data of de Jong et al. (2010), and shows that the BOKS field is typical of fields observed through the Milky Way's disk.

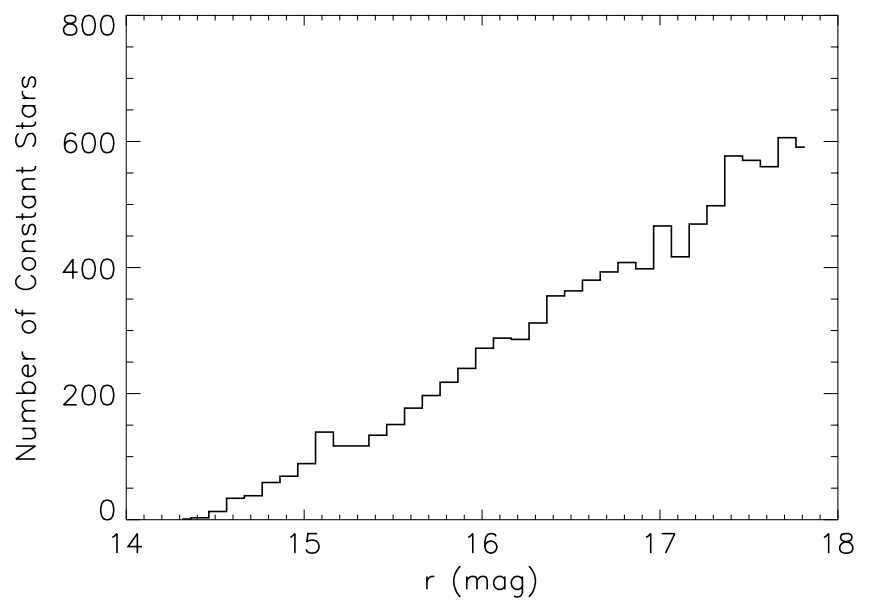

Figure 9. Comparison of the number of photometrically constant stars as a function of magnitude. As expected, the number of photometrically constant stars rises with apparent magnitude since the total number of stars increases, and our ability to search for photometric variations depends strongly on signalto-noise.

a plume around a color of $g-r=0.4-0.6$, corresponding to the main-sequence turn-off for an old ( $>10 \mathrm{Gyr}$ ) stellar population. The BOKS survey combined with the KIC does not go photometrically deep enough to detect the very faint and red low-mass stars in the disk population, which typically appear at $g-r \approx 1.4$ and begin at $r \approx 18.2$. Similar CMDs are found in the work of de Jong et al. (2010, see their Figures 2 and 3) with data taken from SEGUE.

Figure 9 shows a histogram of the number of photometrically constant stars as a function of $r$ magnitude. The number of photometrically constant stars increases roughly linearly up to the final bin $(r=17.9)$. This reflects both the increase in the number of faint stars and our decreased sensitivity to variability for fainter stars.

Some example light curves of stars that show little signs of variability over the timescale of our survey are given in Figure 10. Note that in such long surveys, it is highly likely for a star to suffer at least one, and possibly more, hits from radiation events, such as cosmic rays. 

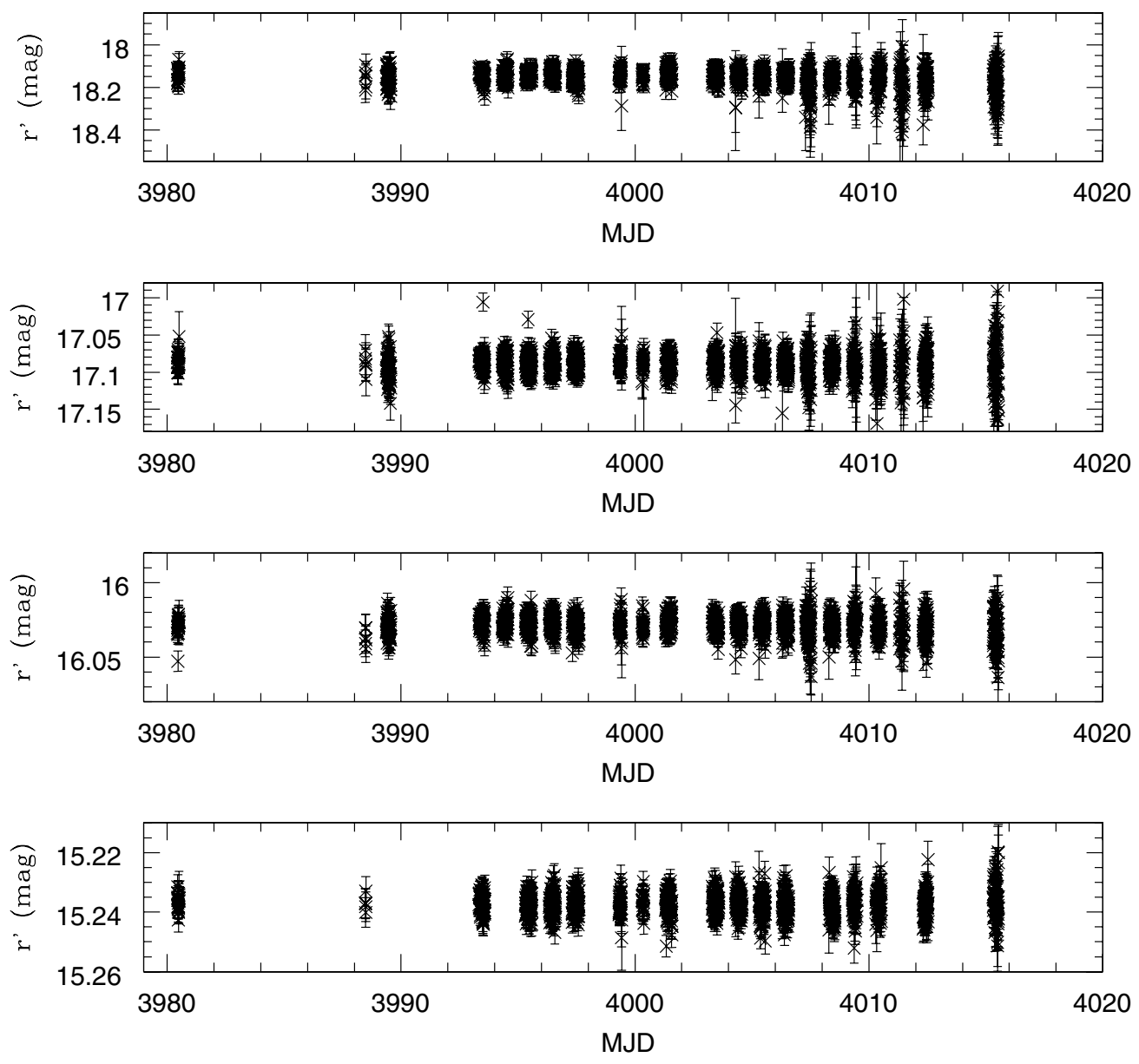

Figure 10. Example of four stars with differing magnitudes that were found to be photometrically constant throughout the survey, using the criteria for variability. From top to bottom the stars are BOKS-33934, BOKS-11505, BOKS-4700, and BOKS-3767. Note that the magnitude axis is substantially different for each star, ranging from $700 \mathrm{mmag}$ for BOKS-33934 down to $50 \mathrm{mmag}$ for BOKS-3767. Many of the most deviant points in each of the light curves are due to radiation events (cosmic rays) hitting the star's aperture.

\subsubsection{Periodic Variable Stars}

In order to test each variable star light curve for periodicity we applied the Lomb-Scargle method (Lomb 1976; Scargle 1982), as described by Press et al. (1992). The algorithm produces a periodogram giving probabilities for sinusoidal signals in the light curves over a range of periods from our minimum sampled period of $1 / 60$ days $(0.4 \mathrm{hr})$, set by the spacing of consecutive exposures, up to 40 days, the maximum possible period spanned by the entire data set. We identified stars as possible periodic variables if (1) OPTICSTAT returned a false probability of periodic variability of less than $1 \times 10^{-4}$, (2) the amplitude was less than $2.5 \mathrm{mag}$, and (3) the average magnitude was brighter than $r=18.5$. This yielded a large but manageable list of candidates. We then visually inspected the light curves of these candidates after phasing them to the best-fit period. Only those light curves that had clear periodic signals and whose light curves showed no sign of systematic effects were accepted as potential periodic variable stars. In order to reduce the effects of aliasing, we also removed from our sample any sources that had periods of $1 \pm 0.025$ days, though some of these objects may be genuine variable stars.

We found that 776 stars from our variable sub-sample had periodic signals that ranged from $\sim 0.2$ day to $\sim 33$ days. The coordinates, mean $r$ magnitudes, determined period and amplitude, and the corresponding KIC information are presented in Table 3. Additionally, we have classified the periodic variables into approximate types, which are also presented in Table 3. Of these objects, $78(\approx 10 \%)$ show variability amplitudes larger than $\sim 0.1$ mag. Another 235 objects $(\approx 30 \%)$ have periodic amplitudes of $1 \%-3 \%$ and periods of 1-3 weeks, which are consistent with rotational modulation due to star spots. A significant number $(N=93 ; 12 \%)$ of the periodic variables remaining have periods less than 2 days and photometric amplitudes less than 0.05 . These short period low amplitude variables are likely to be pulsational variables such as $\delta$ Scuti stars (Breger 2000).

We next compared the properties of the periodic variable stars as a function of period, stellar color, and magnitude, as these distributions give insight on stellar properties in general. It is well known that there are systematic changes in the fraction and amount of stellar variability across the H-R diagram (Eyer \& Mowlavi 2008; Ciardi et al. 2011, and references therein), but the precise distributions are still under debate. Second, studies of these distributions allow us to compare our results with other high precision variability surveys and provide confidence that the survey is valid. Figure 11 shows the overall distribution of periods in our sample of periodic variables. The most notable features of this plot are the large number of stars with periods longer than 10 days and the peak in the period distribution near periods of $\sim 1$ day. Despite our attempts to remove them, this peak strongly suggests that a fraction of these variable stars 
Table 3

Periodic Variable Candidates

\begin{tabular}{lcccccrcc}
\hline \hline $\begin{array}{l}\text { BOKS } \\
\text { ID }\end{array}$ & $\begin{array}{c}\text { KIC } \\
\text { ID }\end{array}$ & $\begin{array}{c}\text { R.A. } \\
\text { (J2000) }\end{array}$ & $\begin{array}{c}\text { Decl. } \\
(\mathrm{J} 2000)\end{array}$ & $\begin{array}{c}r \\
(\mathrm{mag})\end{array}$ & $\begin{array}{c}g-r \\
(\mathrm{mag})\end{array}$ & $\begin{array}{c}\text { Period } \\
(\text { days })\end{array}$ & $\begin{array}{c}\text { Amplitude } \\
(\mathrm{mag})\end{array}$ & \begin{tabular}{c} 
Type/Comments \\
\hline 00413
\end{tabular} \\
\hline 9591127 & 193225.250 & 461238.64 & 16.08 & 1.49 & 1.03 & 0.022 & Delta Scuti \\
00691 & 9652324 & 193227.362 & 462230.47 & 17.00 & 0.61 & 1.03 & 0.030 & Delta Scuti \\
00869 & 9652354 & 193229.766 & 461806.48 & 15.57 & 0.64 & 1.03 & 0.008 & Unknown \\
01418 & 9652406 & 193235.831 & 461946.78 & 14.95 & 0.82 & 28.07 & 0.005 & Rotational modulation \\
01746 & 9591279 & 193239.800 & 461312.29 & 15.38 & 0.66 & 7.11 & 0.013 & Rotational modulation \\
01804 & 9468183 & 193241.141 & 460536.53 & 15.10 & 0.76 & 17.86 & 0.005 & Rotational modulation \\
02055 & 9468216 & 193244.107 & 460132.61 & 17.83 & 1.42 & 4.51 & 0.111 & Rotational modulation \\
02223 & 9468233 & 193245.976 & 460126.53 & 16.27 & 1.22 & 22.47 & 0.02 & Rotational modulation \\
02327 & 9591337 & 193245.886 & 461614.55 & 17.54 & 0.86 & 29.55 & 0.086 & Unknown \\
02867 & 9468296 & 193252.784 & 460312.92 & 15.24 & 0.86 & 5.67 & 0.014 & Unknown \\
\hline
\end{tabular}

(This table is available in its entirety in machine-readable and Virtual Observatory (VO) forms in the online journal. A portion is shown here for guidance regarding its form and content.)

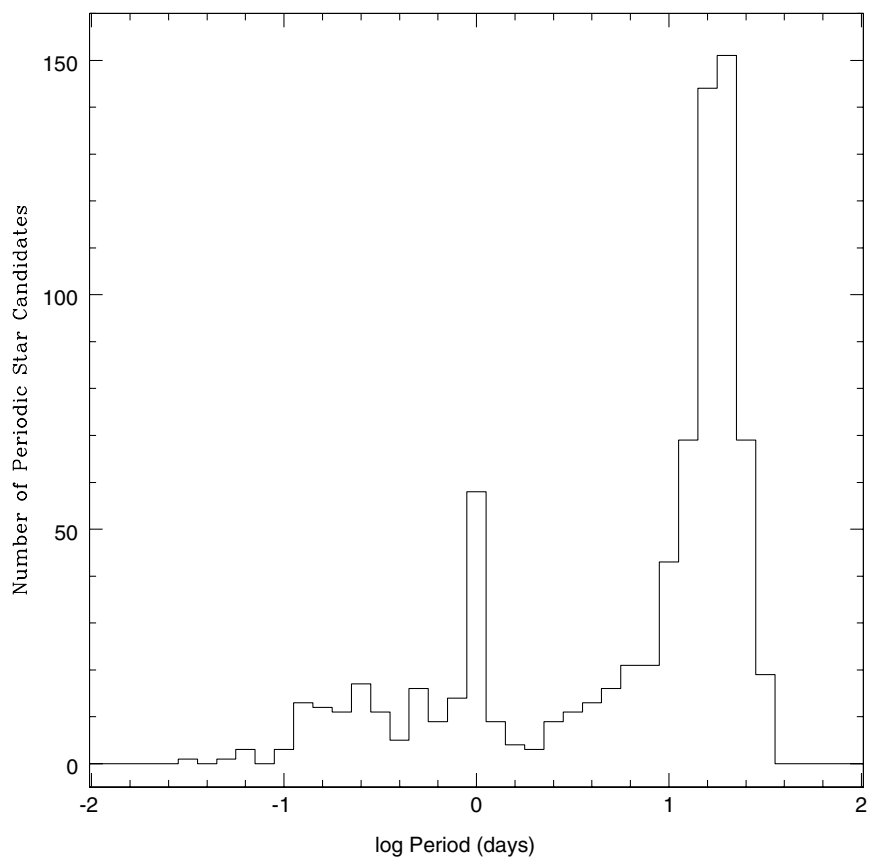

Figure 11. Histogram of the number of periodic stars in our sample with $r$ magnitudes brighter than 18.5 vs. the logarithm of the derived period. Roughly half of the periodic stars we have identified have periods longer than 10 days. There is also a noticeable spike in the distribution near 1 day, suggesting that some of the periods we have identified are due to aliasing near the cadence of our observations, despite the fact we removed objects with periods of $1 \pm$ 0.025 days (Section 5.2.2).

have derived periods that are reflective of aliasing due to our observational window function, rather than their actual period. Additionally, the stars found to be periodic with the longest periods only have one or two measured periods, and the periodfinding algorithm may have mistakenly flagged these objects, when in fact, they may not be periodic. More observations of these particular stars will be required to fully address this issue.

In contrast to the number of photometrically constant stars, a histogram of the number of periodic variables versus $r$ magnitude, which is plotted in Figure 12, shows a steep rise in the number of periodic variables between $r \sim 14$ and $r \sim 16$, which is followed by a steep decline in the number of periodic stars fainter than $r \sim 17$. This decline is due to the rapid loss of photometric sensitivity to low amplitude variations for the fainter stars. Figure 13 shows a histogram of the number of periodic variables versus $g-r$ color. The overall shape of this

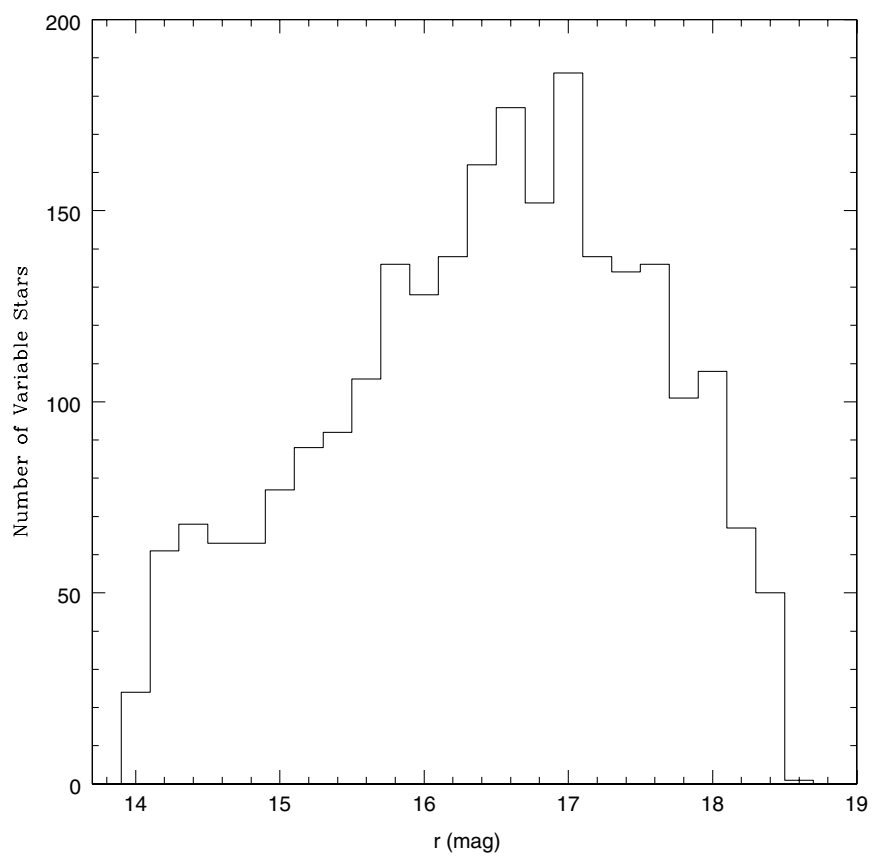

Figure 12. Histogram of the number of periodic variable stars as a function of $r$ magnitude. The number of variable stars detected rises with increasing magnitude down to $r \sim 16$. The number of detected variables drops rapidly between $r \sim 17$ and $r \sim 19$. This reflects the loss of sensitivity to low amplitude variables, such as stars with rotational modulation, as the photometric uncertainties increase.

distribution is very similar to the color-magnitude distribution for constant stars (see Figure 8). There may be a small excess of the bluest stars $(g-r<0.3)$ and the reddest stars $(g-r>1.0)$, but it is unclear whether this is statistically significant. In contrast, Figure 14 plots the period of periodic stars versus their $g-r$ color. Most variables have colors that are similar to those of constant stars regardless of period. There seems to be a small excess of stars with $0 \leqslant g-r \leqslant 0.5$ among the periodic variables with the shortest periods ( $P \lesssim 1$ day).

Figure 15 presents the distribution of amplitude for the periodic variables within the BOKS survey. As has been previously seen (Howell et al. 2005), the number of variable stars increases as the amplitude of variability decreases. The dashed line indicates the power-law model of variability found by Tonry et al. (2005), which has a slope of -2 . The fit is in good agreement, giving further evidence to applicability of this model. 


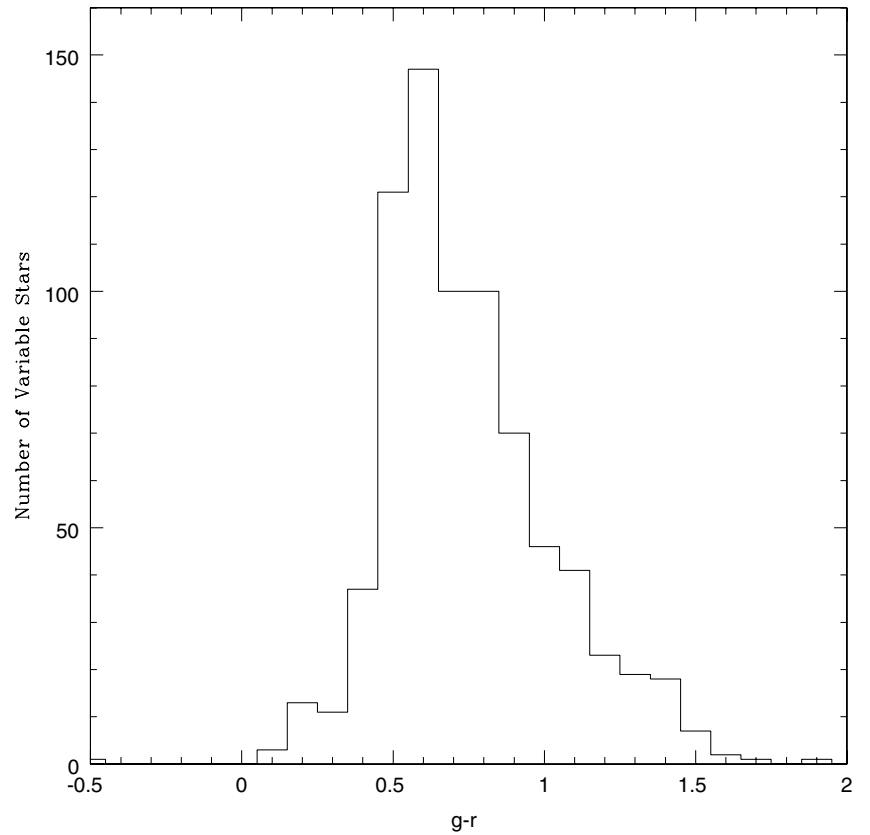

Figure 13. Histogram of the number of periodic variables with $r$ brighter than 18.5 vs. $g-r$ color. This distribution has a single peak near $g-r=0.6$ and a tail that extends to $g-r \sim 1.6$. This is similar to the color-magnitude diagram of non-variable stars (Figure 8), except that there may be a small excess of variables near $g-r=0.2$.

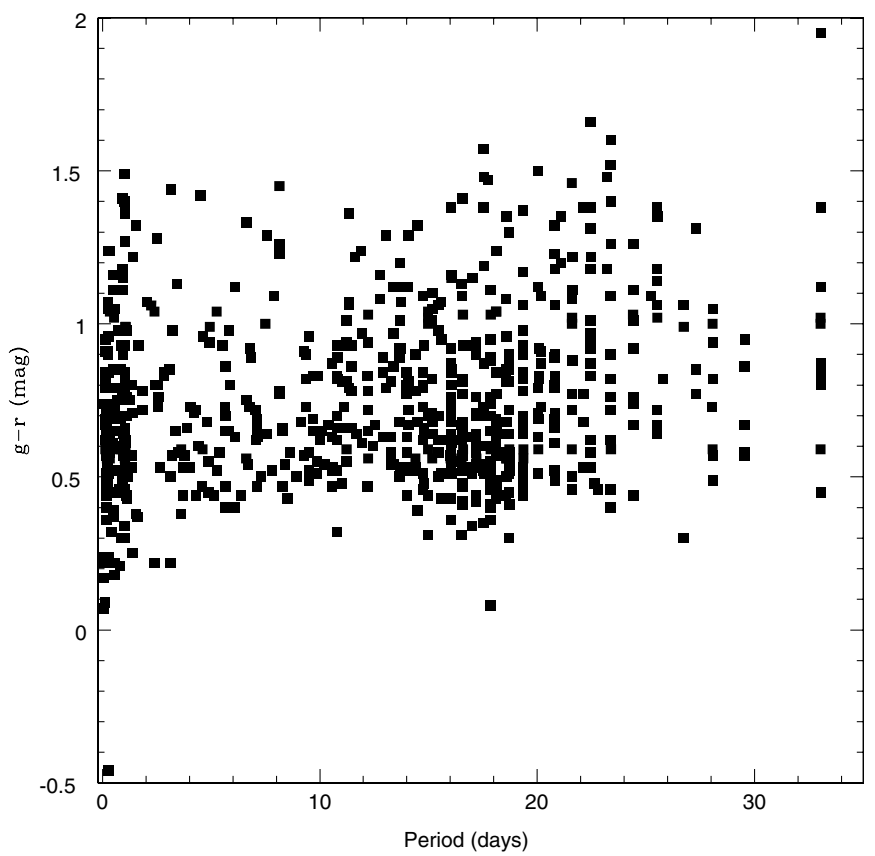

Figure 14. Plot of the period of the periodic variable stars vs. their $g-r$ color. As can be clearly seen, most variables have colors that are similar to those of photometrically constant stars at all periods. For the shortest period stars, $P \lesssim$ 1 day, there seems to be a small excess of stars with $0 \leqslant g-r \leqslant 0.5$.

Although a full accounting of the BOKS periodic variable stars would be soporific, we briefly discuss some of the more interesting stars here, and we discuss two extreme examples in Section 5.4. Among the periodic stars with derived amplitudes larger than $10 \%$, we find at least 10 clear pulsational variables. An example light curve of this type of variable star is plotted in Figure 16. We have also detected two probable RR Lyrae stars within the field, with approximate periods of 0.53 and 0.56 days,

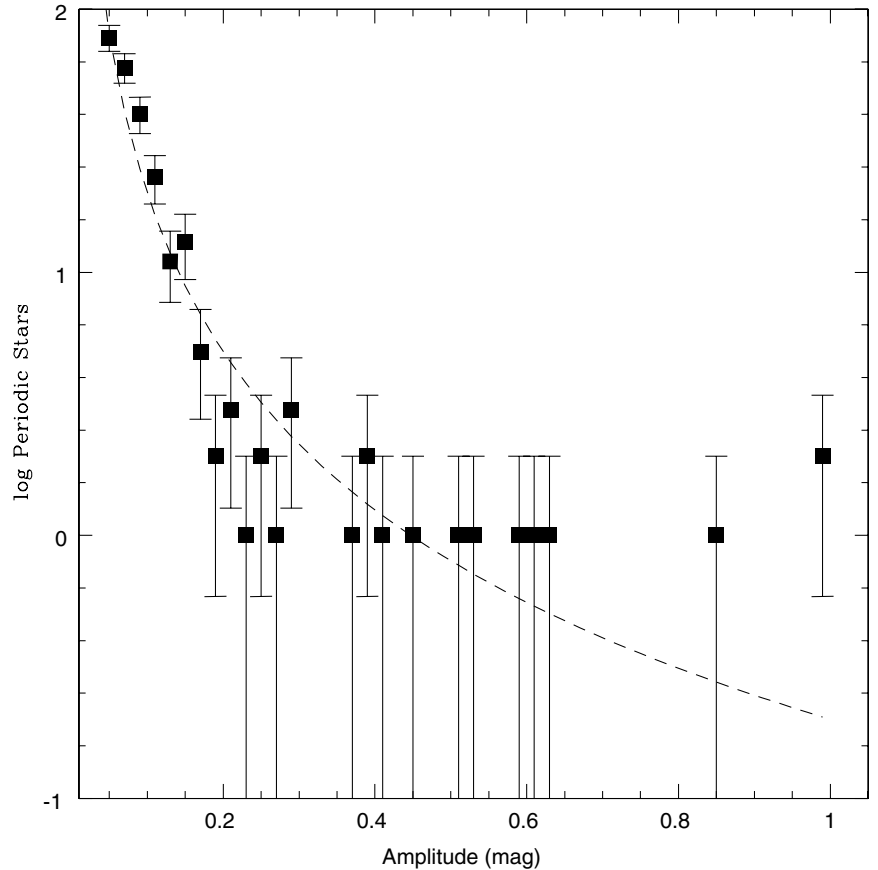

Figure 15. Distribution of periodic variables found in BOKS, as a function of photometric amplitude. The dashed line is the predicted function of variability from Tonry et al. (2005). The error bars are the Poisson uncertainties for each bin.

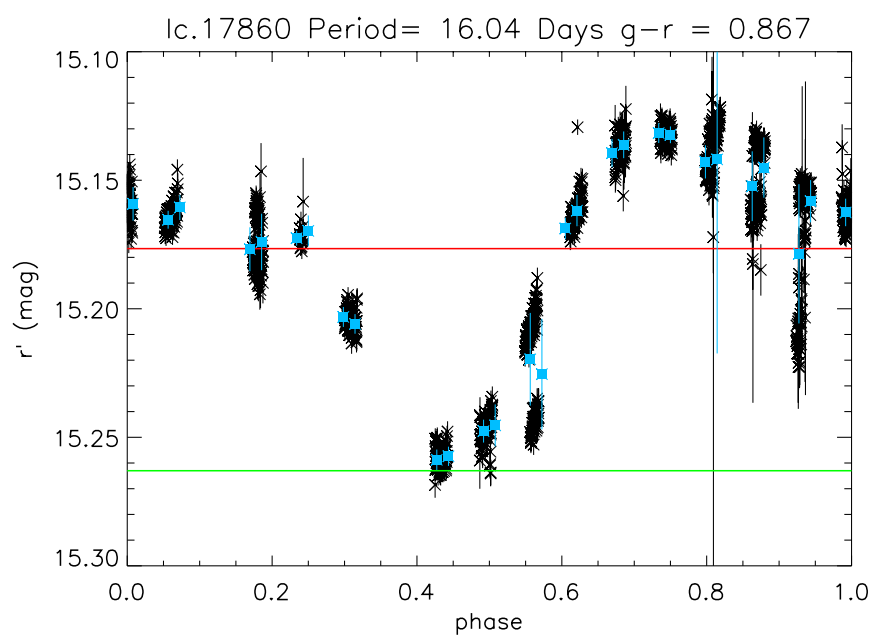

Figure 16. Phased light curve: an example of a pulsating variable star from our survey. The black points are the individual photometric measurements (and error bars). The blue squares are weighted averages calculated for every 26 points of the phased light curve. The red horizontal line at $r \approx 15.18$ is the weighted average $r$ magnitude of the entire light curve. The green horizontal line at $r \approx 15.26$ is the magnitude listed for the star in the Kepler Input Catalog (KIC). (A color version of this figure is available in the online journal.)

respectively. A light curve of one of these objects is plotted in Figure 17.

There are at least 32 eclipsing/contact binaries within the BOKS survey field, with periods varying from 0.13 to 6.10 days. Figures 18 and 19 give examples of two of these systems. From visual inspection of the light curves, the majority of these systems are of the W Ursae Majoris subtype, as would be expected (Hoffman et al. 2009). However, we have also found at least eight Algol-type binary systems. 


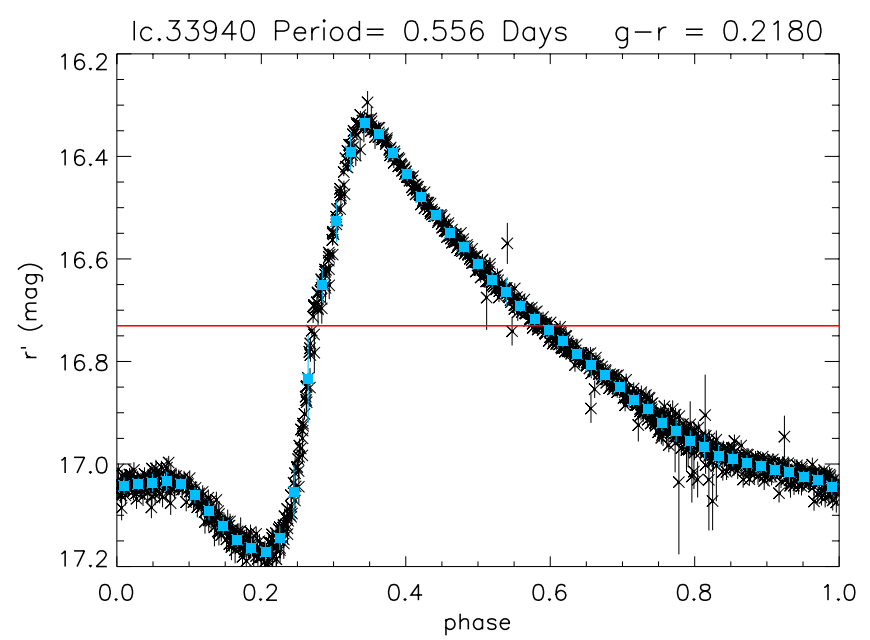

Figure 17. This phased light curve is one example of an RR Lyrae star found within the BOKS field. From visual inspection, the star appears to be of the RRab subtype. The symbols in this plot have identical properties to those found in Figure 16, except that only the mean magnitude is displayed as a horizontal line.

(A color version of this figure is available in the online journal.)

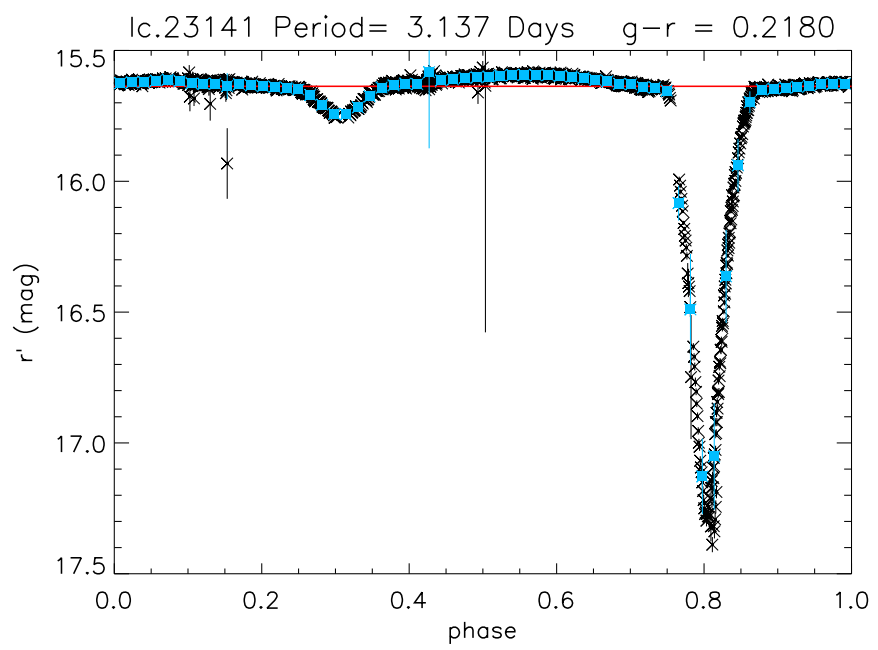

Figure 18. Phased light curve: an example of an eclipsing binary of the Algol type. The symbols are the same as in Figure 17.

(A color version of this figure is available in the online journal.)

\subsection{Exoplanet Transit Candidates}

A primary goal of BOKS is to search for any signs of transiting extrasolar planets in the data set. To search the light curves for transits, we used a simple test to find and flag light curves containing at least one occurrence of a diminution in the relative flux with parameters specified below. The algorithm searched a light curve for time intervals when the mean magnitude is statistically significantly fainter than in the preceding and following data points, in other words, an inverse "top-hat" light curve. The algorithm stepped through time in each light curve testing a grid of possible interval starting and ending times and durations and reported back the most significant possible transit found for each light curve. A probability of significance is determined for all light curves for each possible transit by calculating a Student's $t$-test (Press et al. 1992) statistic comparing the mean magnitude during transit to the mean magnitude of combined pre- and post-transit data points. Those light curves with the most significant probabilities, specifically

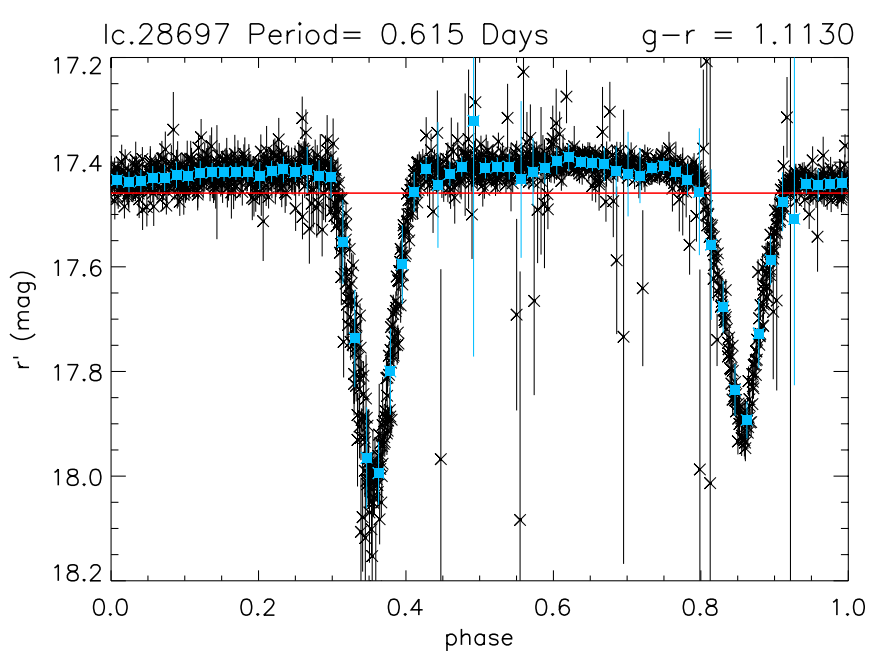

Figure 19. Example of an Algol-type eclipsing variable star that passed the initial OPTICSTAT tests for a transiting extrasolar planet, but was removed after visual inspection. The very deep $(0.6 \mathrm{mag})$ and V-shaped primary eclipse and the clear presence of a secondary eclipse rules this object out as a planetary transit system. The symbols are the same as in Figure 17.

(A color version of this figure is available in the online journal.)

those that have a formal false probability less than $1 \times 10^{-16}$, were then subjected to further inspection. In order to avoid detecting too many spurious light curve fluctuations as transits, we next placed further restrictions on the events reported by the transit-finding algorithm. First, we searched for transit durations only between 1 and $12 \mathrm{hr}$. Second, we placed limits on the depth of transit that merited further study. Large planets orbiting all but the smallest $M$ dwarf stars will result in transit depths of $0.5 \mathrm{mag}$ or less. We therefore removed all possible transits that had depths larger than $0.5 \mathrm{mag}$.

For the purpose of completeness, we decided to use all of the stars in this analysis. It is extremely unlikely that faint stars will show a genuine transit event, but including these objects in the search allows us to test for other systematic effects in the algorithms. The entire BOKS sample contains 54,687 light curves that were all analyzed using OPTICSTAT. Using our detection limit described above, OPTICSTAT identified approximately 1,445 light curves with evidence of transit events.

Each of these 1445 light curves was then inspected by eye with careful attention given to additional criteria. We required at least two data points during transit and at least two data points in both the pre- and post-transit light curve, and we also required that the pre- and post-transit data points be separated by no more than $24 \mathrm{hr}$ from the time of ingress or egress. The transit search algorithm used by OPTICSTAT searched for an inverted "top hat" shape in the light curve. However, there are a number of types of variability that can lead to a "top hat" shape that must be eliminated through a visual inspection of the light curve. Extrasolar planets will cause transits that have a flat-bottomed appearance, so any sharp-bottomed transits were rejected from further consideration. It is not possible with our data set to observe secondary transits resulting from the planet passing behind its star, so any light curve showing secondary transits was also removed from further study. Finally, in order to do follow-up observations we required that the light curve have at least two transit events. We require this to confirm the first transit-event and to obtain an accurate determination of the planet period before any follow-up observations are planned. 

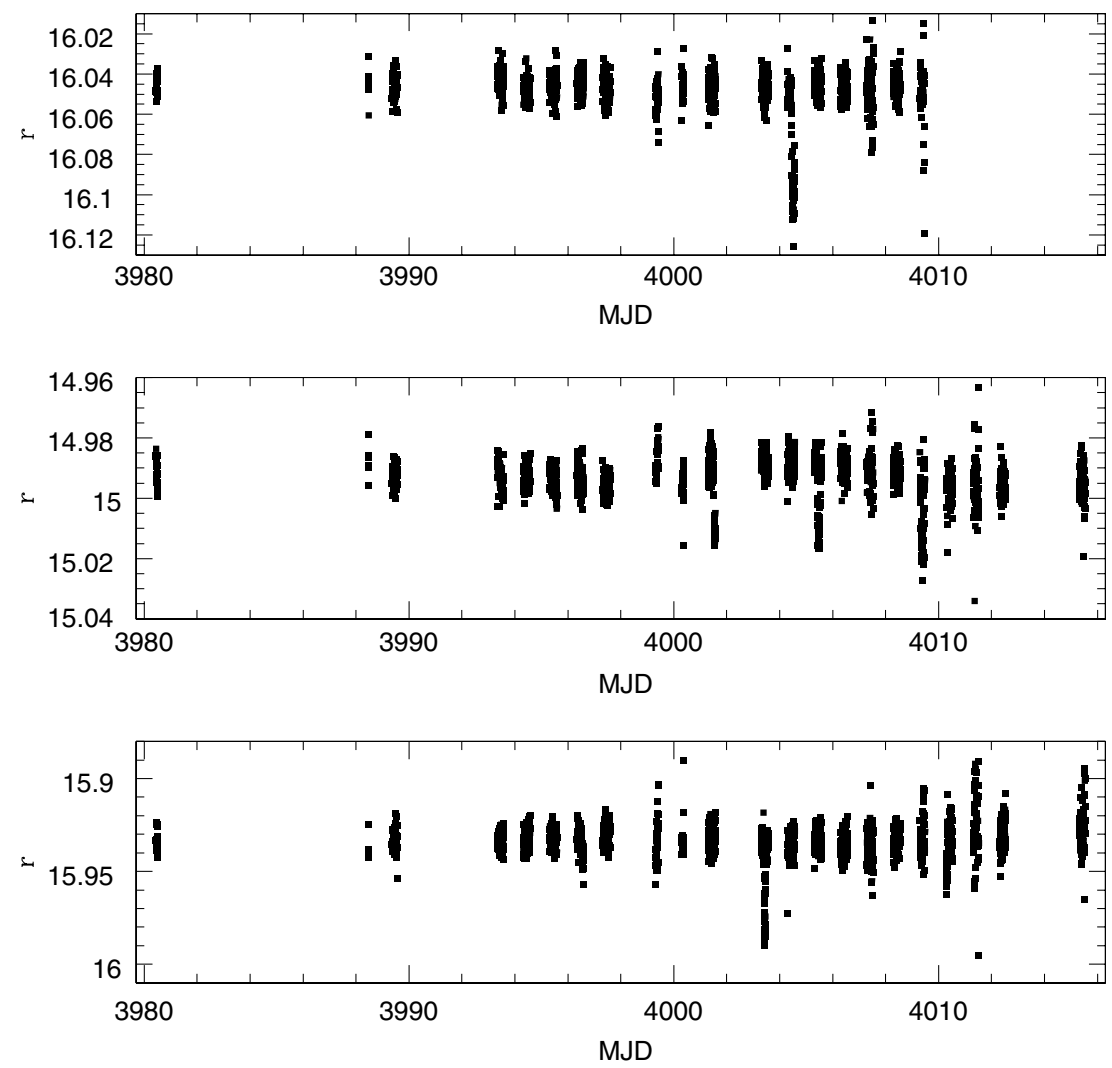

Figure 20. Three highest quality extrasolar candidates from the BOKS survey. In order from top to bottom, they are BOKS-45069, BOKS-40959, and BOKS-52481. The error bars are omitted for clarity, but typical $2 \sigma$ uncertainties for these three light curves are $0.012,0.014$, and 0.012 mag, respectively.
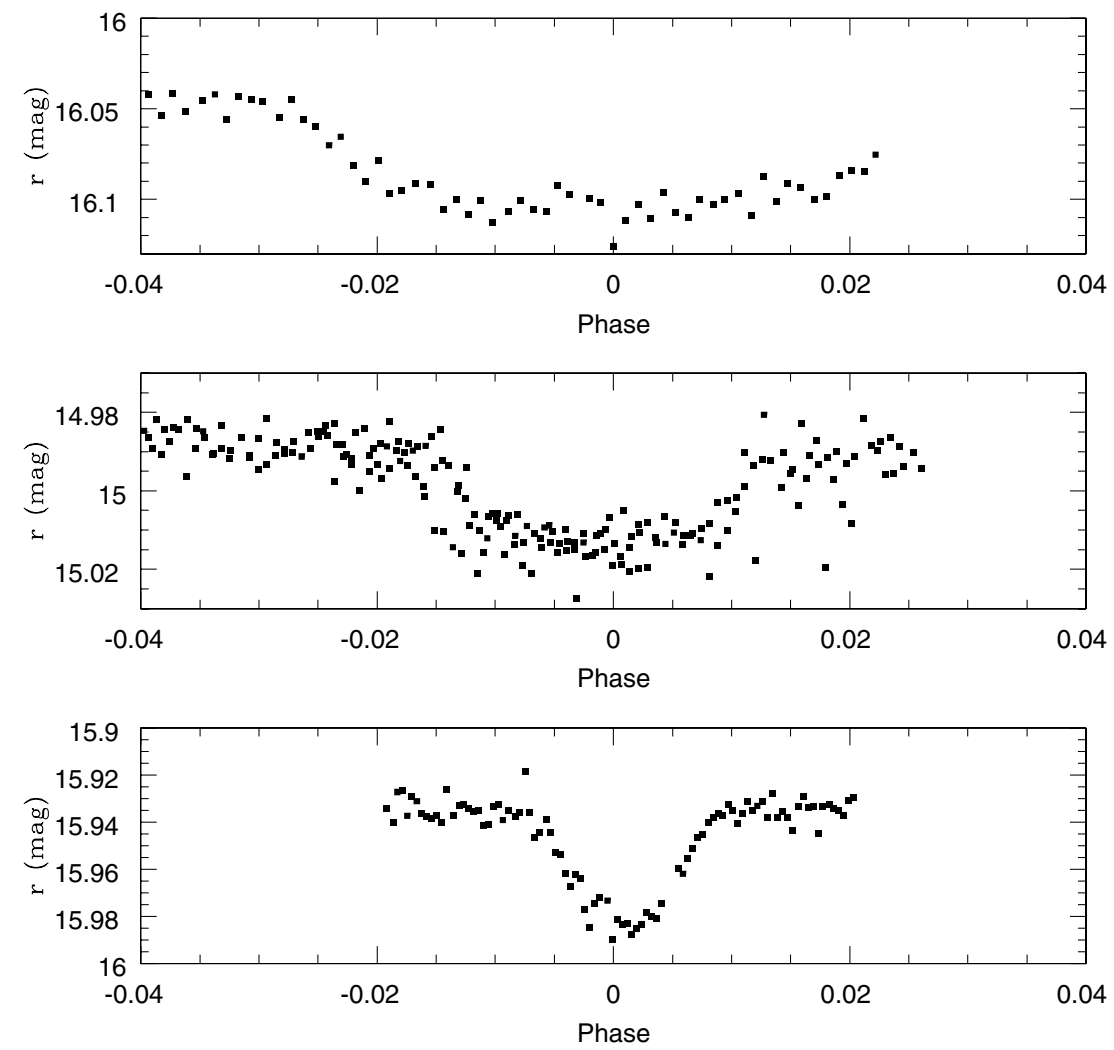

Figure 21. Light curves of our three highest quality extrasolar candidates near the transiting phase. In order from top to bottom, they are BOKS-45069, BOKS-40959, and BOKS-52481. The error bars are omitted for clarity, but typical $2 \sigma$ uncertainties for these three light curves are $0.012,0.014$, and 0.012 mag, respectively. 


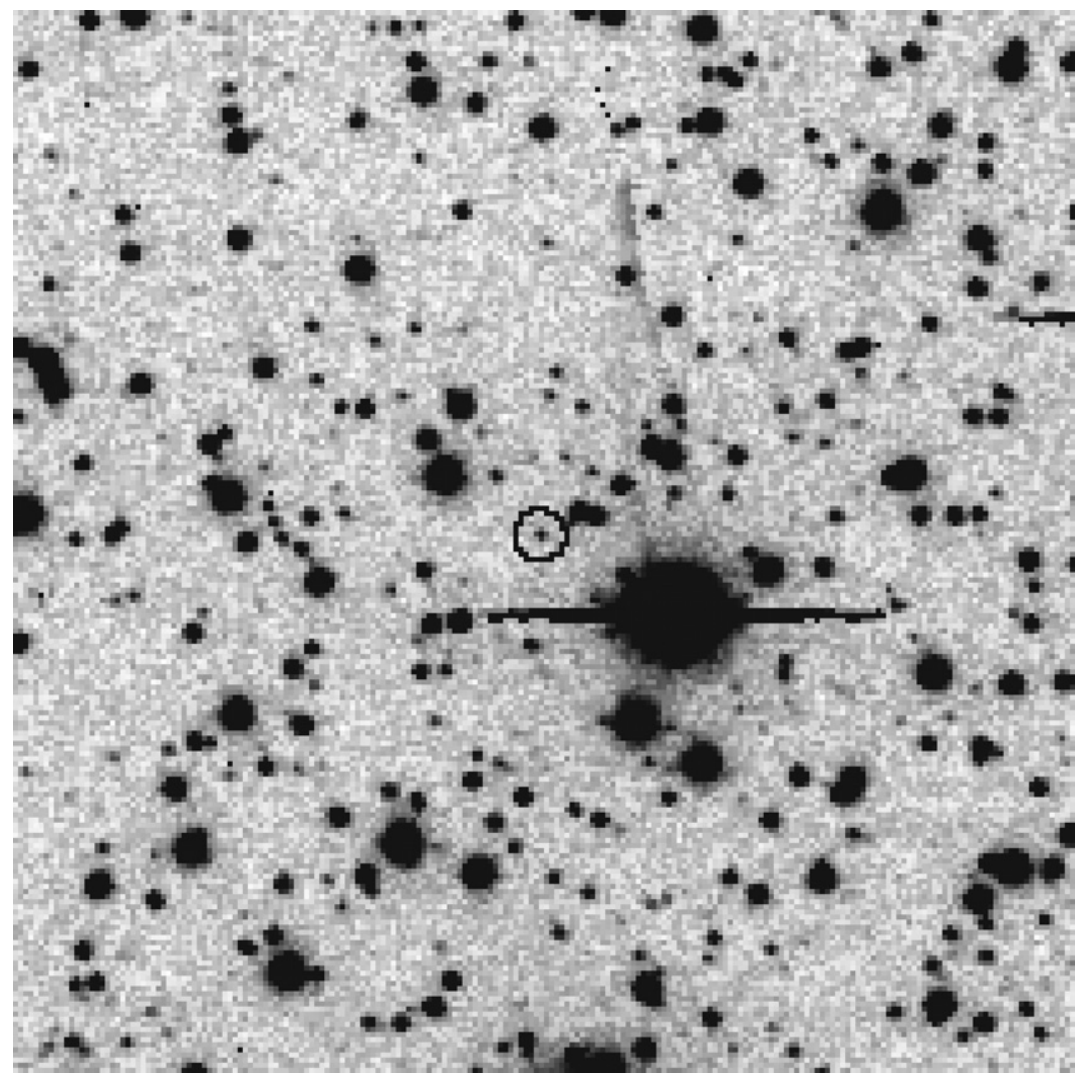

Figure 22. Finding chart of a dwarf nova found by the BOKS survey. This finding chart is $5^{\prime} \times 5^{\prime}$ in size, and north is up and east is to the left on this image. The object, known as BOKS 45906, is located at $\alpha=19^{\mathrm{h}} 40^{\mathrm{m}} 16^{\mathrm{s}} .22, \delta=+46^{\mathrm{d}} 32^{\mathrm{m}} 48^{\mathrm{s}} .23$, and is centered in this image, surrounded by a circle for reference. The emission north by northwest of this candidate is a Schmidt ghost and is unrelated to the source. See Section 5.4 for a discussion of this object.

Table 4

BOKS Exoplanet Candidates

\begin{tabular}{|c|c|c|c|c|c|c|c|c|}
\hline BOKS ID & KIC ID & $\begin{array}{l}\text { R.A. } \\
\text { (J2000) }\end{array}$ & $\begin{array}{c}\text { Decl. } \\
\text { (J2000) }\end{array}$ & $\begin{array}{c}r \\
(\mathrm{mag}) \\
\end{array}$ & $\begin{array}{l}g-r^{\mathrm{a}} \\
(\mathrm{mag})\end{array}$ & $\begin{array}{l}\text { Period } \\
\text { (days) }\end{array}$ & $\begin{array}{l}\text { Eclipse Depth } \\
\text { (mag) }\end{array}$ & Notes \\
\hline 40959 & 9595827 & 193927.667 & 461709.23 & 15.1 & 0.63 & 3.9 & $0.02 \pm 0.01$ & \\
\hline 45069 & 9838975 & 194008.003 & 463601.22 & 16.1 & 0.73 & 2.6 & $0.04 \pm 0.01$ & \\
\hline 52481 & 9597095 & 194118.802 & 461606.00 & 15.9 & 0.63 & 7 & $0.05 \pm 0.01$ & Period approximate \\
\hline
\end{tabular}

Note. ${ }^{\text {a }}$ For reference, the Sun is believed to have a $g-r$ color of $0.44 \pm 0.02$ (Bilir et al. 2005; Rodgers et al. 2006).

The light curve shown in Figure 19 was incorrectly identified by OPTICSTAT as a transit candidate, but was easily removed by our manual inspection criteria: the transits in this light curve are too deep ( $\approx 0.6 \mathrm{mag}$ ), sharp-bottomed, and there is an obvious secondary transit. Nearly all of the transit candidates detected by OPTICSTAT were rejected using the simple requirements we have outlined.

At the end of our analysis we were left with three exoplanet candidates: BOKS-45069, BOKS-40959, and BOKS-52481. Some basic properties of these candidates are given in Table 4 , and a plot of their light curves is given in Figure 20. For each of the candidates, we then determined an approximate period by phasing the light curve to the best-fitting phase. In the case of BOKS-52481, we detected one full transit and only a portion of another transit, so the measured period is substantially less certain than the other two candidates. We also determined an approximate transit depth by averaging the closest 40 light curve points immediately before and after the transit to obtain a baseline. We present an example of each transit in Figure 21.

The properties derived are similar to other ground-based transit detections, but a detailed analysis, including follow-up spectroscopic and imaging observations of these candidates, is presented in Howell et al. (2010).

\subsection{Two Specific Cases of Stellar Variability}

During any large area photometric survey, there is the potential of discovering unusual to rare objects. In the case of BOKS, we detail here two unusual variable stars that we have found in the survey.

\subsubsection{BOKS -45906}

The first object, whose location is displayed in Figure 22, is known as BOKS-45906 (KIC 9778689). On the first two clear nights of our survey (MJD 3980 and 3988), the star had a mean magnitude of $r \approx 20$, though there were clear signs of variability of up to a magnitude in amplitude. The star continued to vary on both nightly and intra-nightly timescales. Then, between MJD 4004 and 4005, the star had an eruption, reaching a maximum of $r=16.6 \pm 0.01$ on MJD 4006. It then declined in flux, returning to the approximate quiescent flux level on MJD 4009. The overall light curve is plotted in 


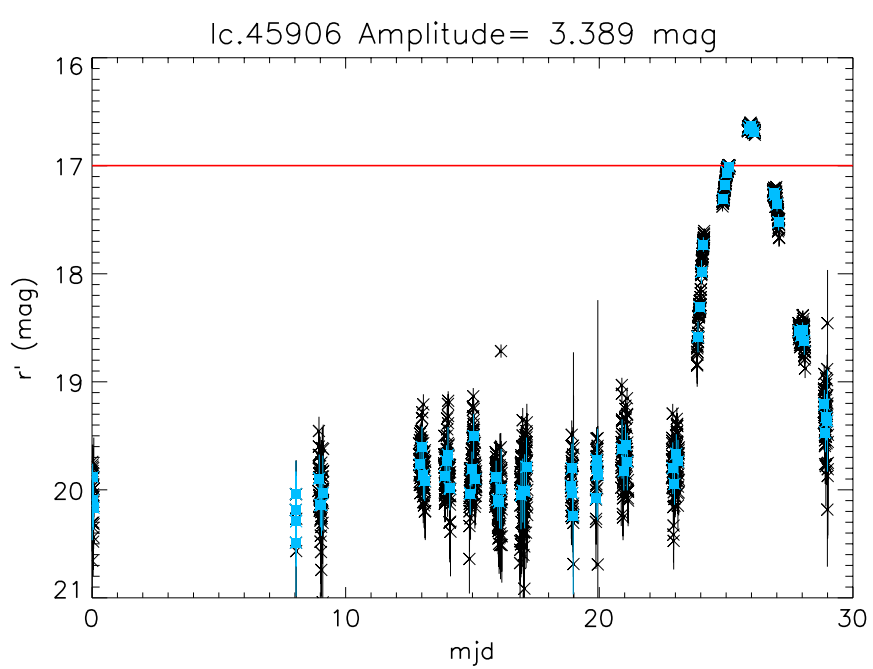

Figure 23. Light curve of a highly variable star found by the BOKS survey. This star, BOKS-45906, underwent a large photometric outburst, which lasted approximately 5 days. This behavior is typical of dwarf novae (see Section 5.4 for discussion).

(A color version of this figure is available in the online journal.)

Figure 23. From the light curve, it is likely that this star is a newly discovered cataclysmic variable of the dwarf nova subtype. There is scientific interest in the light curves of similar objects, both before and after eruption (Robinson 1975; Collazzi et al. 2009; Schaefer et al. 2010); therefore additional photometric monitoring of this source may be helpful.

\subsubsection{BOKS-53856}

The second interesting variable we have found is a blue star in the BOKS survey (a finding chart is displayed in Figure 24) known as BOKS-53856. From comparison to the KIC78, it has a measured color of $g-r=-0.46$, making it the bluest stellar source in our field. Analysis of its light curve indicates periodic variability, with a period of 0.255 days, though of an unusual nature. The phased light curve is presented in Figure 25.

We obtained a $900 \mathrm{~s}$ spectrum of BOKS-53856 using the Kitt Peak $2.1 \mathrm{~m}$ telescope and the GoldCam spectrograph on UT 2008 June 26. We used the $3001 \mathrm{~mm}^{-1}$ grating (no. 32) with a 1 arcsec slit to provide a mean spectral resolution of $2.4 \AA$ across the full wavelength range. The spectra were reduced in the normal manner with observations of calibration lamps and spectrophotometric standard stars obtained before and after each sequence and bias and flat frames collected in the afternoon. The final reduced spectrum is displayed in Figure 26.

The most obvious features in the spectrum are the blue continuum and strong Balmer lines, indicating a DA white dwarf type spectrum. In general, blue variables are either of low amplitude and consist of pulsations or as in the case here, show larger variations and may be some sort of interacting binary. J. B. Holberg \& S. B. Howell (2011, in preparation) present a further study of this star.

\section{CONCLUSIONS}

The goal of the BOKS survey was to constrain the amount and nature of variability in a subsection of NASA's Kepler mission field of view. The dedicated observations we conducted of the BOKS field allowed us to observe variability on various

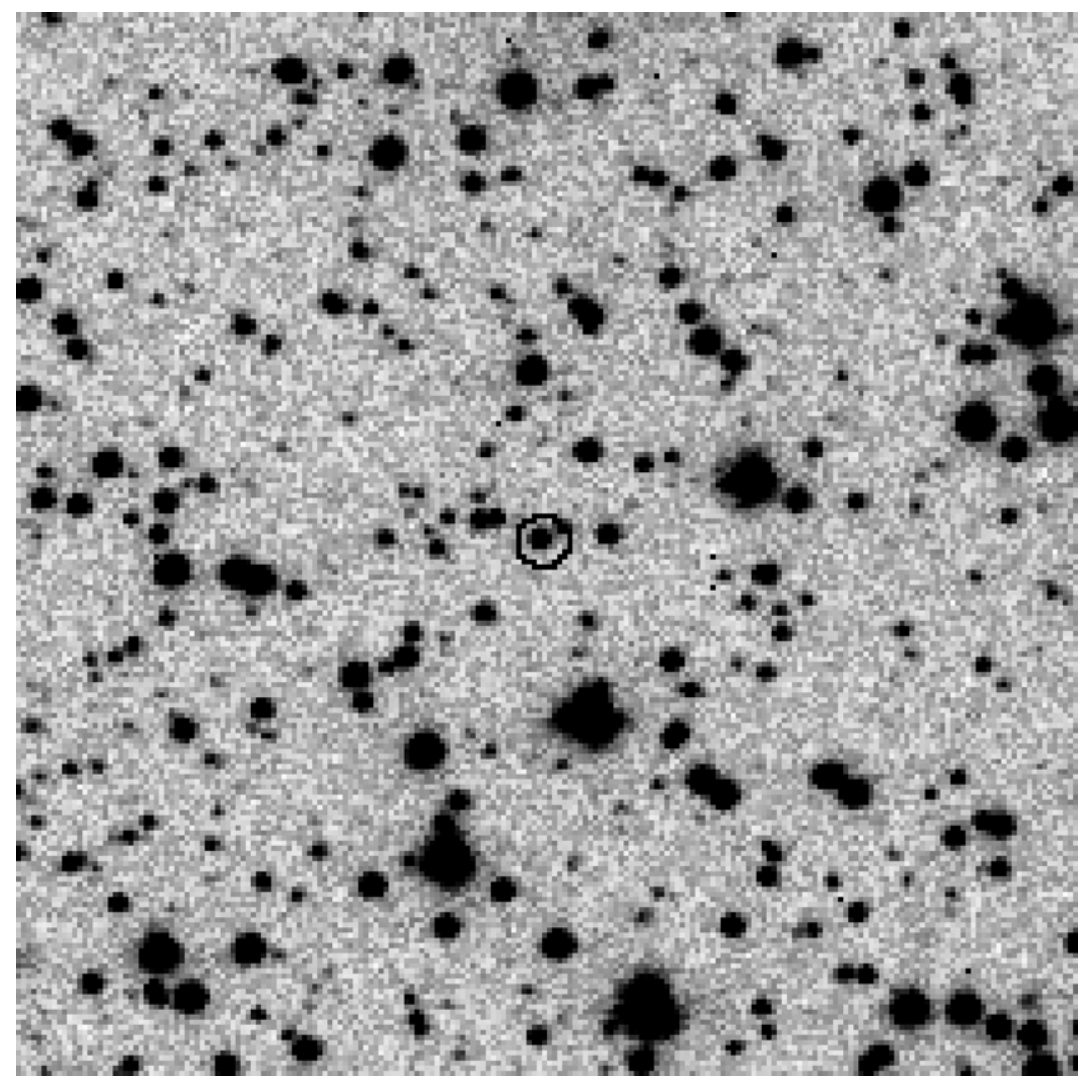

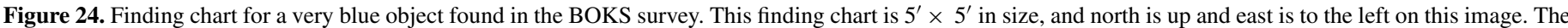

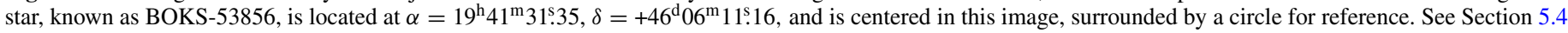
for a discussion of this object. 


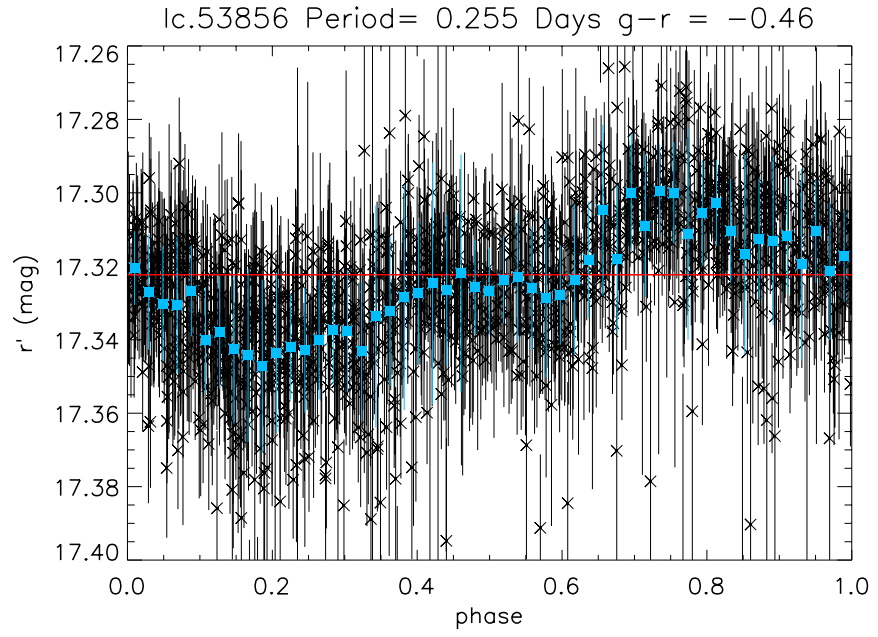

Figure 25. Phased light curve of an extremely blue $(g-r=-0.46)$ object in the BOKS field. The symbols are identical to Figure 17. The light curve shows signs of periodic behavior, but with unusual structure. See Section 5.4 for further discussion.

(A color version of this figure is available in the online journal.)

timescales from a few minutes to many days. The long-term observations also allowed us a reasonable opportunity to search for hot Jupiter exoplanet transits.

Through our preliminary analysis of the variability in the BOKS field we have identified $\sim 2457$ candidate variable stars with 776 candidate periodic variables. Most of this periodic variation can be attributed to rotating, low-mass stars with magnetic activity star/spots. We have also found over $90 \delta$ Scuti stars, over 32 eclipsing binaries and contact binaries, and tens of large amplitude pulsators, such as RR Lyrae stars. Within the BOKS field of view, we have also identified at least three exoplanet candidates, all of which are undergoing observations by the Kepler Mission. The comparison of ground-based and space-based transit observations should be beneficial to many future surveys.

We thank the entire staff of Case Western Reserve University Warner \& Swasey observatory, including Heather L. Morrison, Charles Knox, and Colin Wallace for their invaluable assistance with the Burrell Schmidt. We are also extremely grateful to the observers of the AAVSO who performed many observations of our field concurrently. We thank Richard Wade for some useful discussions. We also thank the anonymous referee for several suggestions that improved the quality of this paper. This research has made use of the WEBDA database, operated at the Institute for Astronomy of the University of Vienna. This publication makes use of data products from the Two Micron All Sky Survey, which is a joint project of the University of Massachusetts and the Infrared Processing and Analysis Center/California Institute of Technology, funded by the National Aeronautics and Space Administration and the National Science Foundation.

Facilities: KPNO:2.1m (Goldcam), CWRU:Schmidt

\section{REFERENCES}

Badiali, M., Catala, C., Fossat, E., Fransden, S., Gough, D. O., Rocca-Cortes, T., \& Schrijver, K. 1994, Observatory, 114, 53

Bakos, G. Á., et al. 2010, ApJ, 710, 1724

Batalha, N. M., et al. 2010, ApJ, 713, L109

Beatty, T. G., \& Gaudi, B. S. 2008, ApJ, 686, 1302

Bilir, S., Karaali, S., \& Tunçel, S. 2005, Astron. Nachr., 326, 321

Borucki, W. J., \& for the Kepler Team 2010a, arXiv:1006.2799

Borucki, W. J., et al. 2010b, Science, 327, 977

Borucki, W. J., et al. 2010c, ApJ, 713, L126

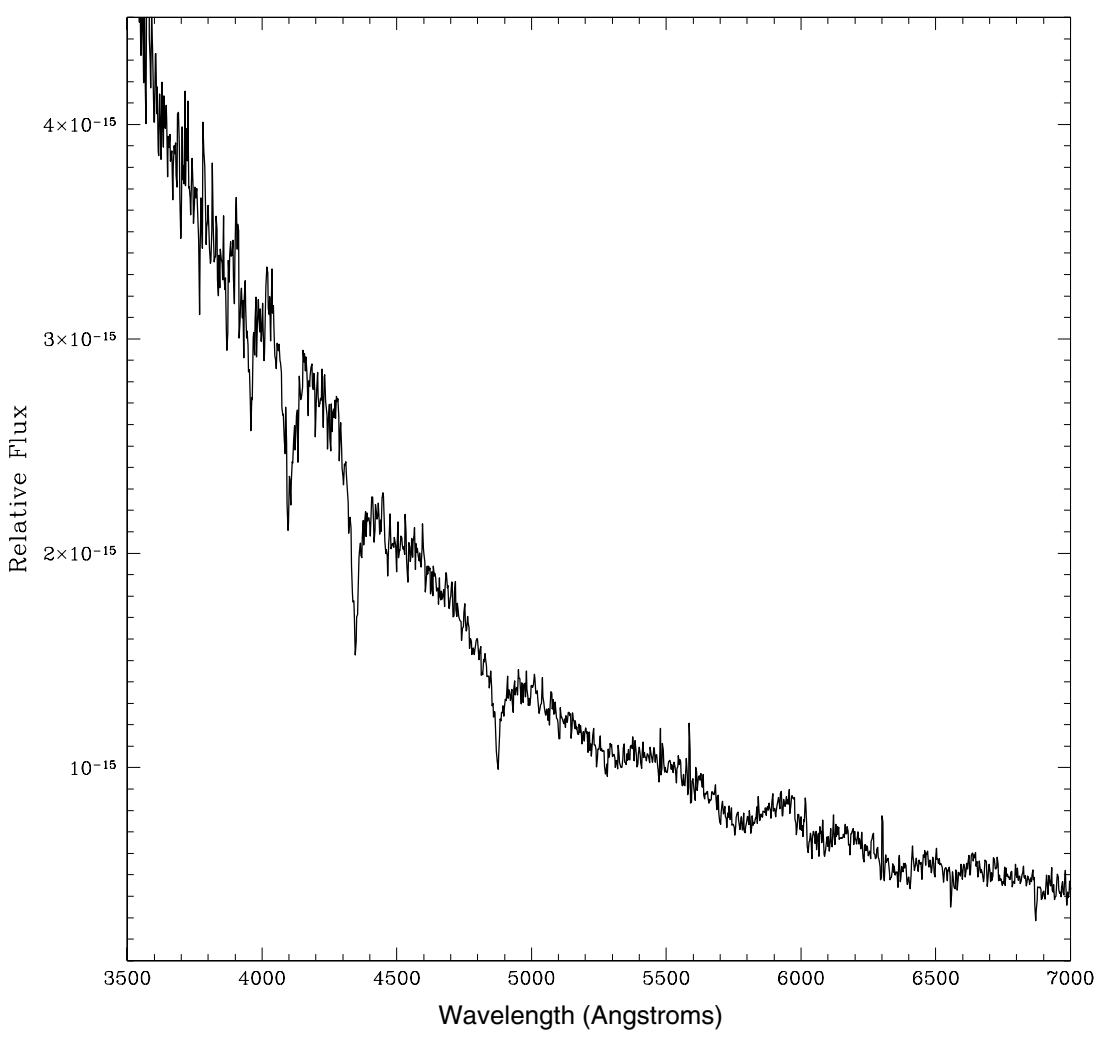

Figure 26. Spectrum of BOKS-53856, a very blue object in the BOKS survey, obtained with the KPNO $2.1 \mathrm{~m}$ telescope. Broad Balmer line features and a blue continuum are clearly visible, suggesting that this object is a white dwarf star. See Section 5.4 for further discussion. 
Breger, M. 2000, in ASP Conf. Ser. 210, Delta Scuti and Related Stars, Reference Handbook and Proc. of the 6th Vienna Workshop in Astrophysics, ed. M. Breger \& M. H. Montgomery (San Francisco, CA: ASP), 3

Burke, C. J., Gaudi, B. S., DePoy, D. L., \& Pogge, R. W. 2006, AJ, 132, 210

Ciardi, D. R. 2011, AJ, 141, 108

Collazzi, A. C., Schaefer, B. E., Xiao, L., Pagnotta, A., Kroll, P., Löchel, K., \& Henden, A. A. 2009, AJ, 138, 1846

de Jong, J. T. A., Yanny, B., Rix, H.-W., Dolphin, A. E., Martin, N. F., \& Beers, T. C. 2010, ApJ, 714, 663

Dunham, E. W., et al. 2010, ApJ, 713, L136

Everett, M. E., \& Howell, S. B. 2001, PASP, 113, 1428

Everett, M. E., Howell, S. B., van Belle, G. T., \& Ciardi, D. R. 2002, PASP, 114,656

Eyer, L., \& Mowlavi, N. 2008, J. Phys. Conf. Ser., 118, 012010

Henden, A. A., Price, A., \& Howell, S. 2006, BAAS, 38, 1126

Hoffman, D. I., Harrison, T. E., \& McNamara, B. J. 2009, AJ, 138, 466

Hood, B., et al. 2005, MNRAS, 360, 791

Howell, S. B. 2008, Astron. Nachr., 329, 259

Howell, S. B., VanOutryve, C., Tonry, J. L., Everett, M. E., \& Schneider, R. 2005, PASP, 117, 1187

Howell, S. B., et al. 2010, ApJ, 725, 1633

Jenkins, J. M., et al. 2010, ApJ, 724, 1108

Kim, S.-L., et al. 2001, A\&A, 371, 571

Koch, D. G., et al. 2010, ApJ, 713, L131

Latham, D. W., et al. 2010, ApJ, 713, L140

Leach, R. W., Beale, F. L., \& Eriksen, J. E. 1998, Proc. SPIE, 3355, 512

Lomb, N. R. 1976, Ap\&SS, 39, 447

Luo, Y. P., Zhang, X. B., Luo, C. Q., Deng, L. C., \& Luo, Z. Q. 2009, New Astron., 14, 584

Mochejska, B. J., et al. 2006, AJ, 131, 1090
Monet, D. G., et al. 2003, AJ, 125, 984

O’Donovan, F. T., et al. 2006, ApJ, 651, L61

Pál, A., et al. 2008, ApJ, 680, 1450

Pont, F., Zucker, S., \& Queloz, D. 2006, MNRAS, 373, 231

Press, W. H., Teukolsky, S. A., Vetterling, W. T., \& Flannery, B. P. 1992, Numerical Recipes in FORTRAN. The Art of Scientific Computing (2nd ed.; Cambridge: Cambridge Univ. Press)

Robinson, E. L. 1975, AJ, 80, 515

Rodgers, C. T., Canterna, R., Smith, J. A., Pierce, M. J., \& Tucker, D. L. 2006, AJ, 132, 989

Rose, M. B., \& Hintz, E. G. 2007, AJ, 134, 2067

Scargle, J. D. 1982, ApJ, 263, 835

Schaefer, B. E., et al. 2010, AJ, 140, 925

Skrutskie, M. F., et al. 2006, AJ, 131, 1163

Steffen, J. H., et al. 2010, ApJ, 725, 1226

Stetson, P. B. 1987, PASP, 99, 191

Sukhbold, T., \& Howell, S. B. 2009, PASP, 121, 1188

Tonry, J. L., Howell, S. B., Everett, M. E., Rodney, S. A., Willman, M., \& VanOutryve, C. 2005, PASP, 117, 281

van Cauteren, P., Lampens, P., Robertson, C. W., \& Strigachev, A. 2005, Commun. Asteroseismol., 146, 21

von Braun, K., Kane, S. R., \& Ciardi, D. R. 2009a, ApJ, 702, 779

von Braun, K., Lee, B. L., Seager, S., Yee, H. K. C., Mallén-Ornelas, G., \& Gladders, M. D. 2005, PASP, 117, 141

von Braun, K., et al. 2009b, in IAU Symp. 253, Transiting Planets, ed. F. Pont, D. Saddelov, \& M. Holman (Cambridge: Cambridge Univ. Press), 478

Welsh, W. F., Orosz, J. A., Seager, S., Fortney, J. J., Jenkins, J., Rowe, J. F., Koch, D., \& Borucki, W. J. 2010, ApJ, 713, L145

Young, A. T. 1967, AJ, 72, 747

Young, A. T. 1993a, Observatory, 113, 41

Young, A. T. 1993b, Observatory, 113, 266 